ANNALES

POLONICI MATHEMATICI

LXXVI.3 (2001)

\title{
Extensions de jets dans des intersections de classes non quasi-analytiques
}

\author{
par Pascal Beaugendre (Orsay)
}

\begin{abstract}
In [3], J. Chaumat and A.-M. Chollet prove, among other things, a Whitney extension theorem, for jets on a compact subset $E$ of $\mathbb{R}^{n}$, in the case of intersections of non-quasi-analytic classes with moderate growth and a Łojasiewicz theorem in the regular situation. These intersections are included in the intersection of Gevrey classes. Here we prove an extension theorem in the case of more general intersections such that every $C^{\infty}$-Whitney jet belongs to one of them. We also prove a linear extension theorem in the case of a compact set with Markov's property. These extensions of jets can be chosen to be real-analytic on $\mathbb{R}^{n} \backslash E$. Then we prove a Łojasiewicz theorem.
\end{abstract}

1. Introduction. On note $E$ un ensemble compact de $\mathbb{R}^{n}$. Une fonction $f$ de classe $C^{\infty}$ sur $\mathbb{R}^{n}$ appartient à l'intersection des classes de Gevrey $\widehat{G}$ lorsque, pour tout $a>0$, on a

$$
\sup _{p \in \mathbb{N}} \sup _{P ; p=|P|} \sup _{x \in \mathbb{R}^{n}} \frac{\left|f^{P}(x)\right|}{p !(p !)^{a}}<+\infty .
$$

Un jet $F$ sur $E$ est la donnée d'une suite $\left(F^{J}\right)_{J \in \mathbb{N}^{n}}$ de fonctions continues sur $E$ à valeurs dans $\mathbb{R}$. Soient $p \in \mathbb{N}$ et $J \in \mathbb{N}^{n}$ un multi-indice de longueur $|J| \leq p ;$ pour tout $(\zeta, x) \in E^{2}$, on pose

$$
R_{\zeta}^{J, p} F(x)=F^{J}(x)-\sum_{K ;|J+K| \leq p} \frac{1}{K !}(x-\zeta)^{K} F^{J+K}(\zeta) .
$$

Un jet $F$, défini sur $E$, appartient à $\widehat{G}(E)$ lorsque, pour tout $a>0$, on a

2000 Mathematics Subject Classification: 26E10, 41A10, 46E10, 46E15.

Key words and phrases: jets, ultradifferentiable functions, Whitney extension theorem, intersection of Carleman classes, Gevrey classes, non-quasi-analytic, Markov's property, analytic extensions, Łojasiewicz theorem. 


$$
\begin{aligned}
& \sup _{p \in \mathbb{N}} \max \left\{\sup _{P ; p=|P| x \in E}\right. \sup _{x \in E} \frac{\left|F^{P}(x)\right|}{p !(p !)^{a}}, \\
&\left.\sup _{J ;|J| \leq p} \sup _{\substack{\zeta, x) \in E^{2} \\
\zeta \neq x}} \frac{\left|R_{\zeta}^{J, p} F(x)\right|}{j !((p+1) !)^{a}|\zeta-x|^{p+1-j}}\right\}<+\infty ;
\end{aligned}
$$

donc $\widehat{G}(E)$ est l'ensemble des jets qui appartiennent à l'intersection des classes de jets de Gevrey sur $E$. Il est clair que, si $f \in \widehat{G}$, alors $\left(f_{\mid E}^{J}\right)_{J \in \mathbb{N}^{n}}$, le jet induit par $f$ sur $E$, appartient à $\widehat{G}(E)$.

Les classes de Gevrey interviennent de façon naturelle dans des problèmes d'équations aux dérivées partielles. J. Chaumat et A.-M. Chollet ont prouvé que leur intersection jouit des mêmes bonnes propriétés que $C^{\infty}\left(\mathbb{R}^{n}\right)$. On pourra consulter [3] et [4] où sont notamment démontrés un théorème d'extension de type Whitney, un théorème de division ainsi qu'un théorème de préparation. L'intersection des classes de Gevrey y est étudiée dans le cadre plus large des intersections à croissance modérée. Ces intersections sont incluses dans l'intersection des classes de Gevrey.

Plus généralement, soit $\phi$ une fonction convexe croissante sur $\mathbb{R}_{+}$. On suppose que $\lim _{t \rightarrow+\infty} \phi(t) / t=+\infty$. Pour tout $a>0$ et tout $p \in \mathbb{N}$, on pose $M_{a p}^{(\phi)}=\exp (\phi(a p))$. Une fonction $f$ de classe $C^{\infty}$ sur $\mathbb{R}^{n}$ appartient à $\widehat{\phi}$ lorsque, pour tout $a>0$, on a

$$
\sup _{p \in \mathbb{N}} \sup _{P ; p=|P|} \sup _{x \in \mathbb{R}^{n}} \frac{\left|f^{P}(x)\right|}{p ! M_{a p}^{(\phi)}}<+\infty .
$$

De même, un jet $F$ défini sur $E$ appartient à $\widehat{J \phi}(E)$ lorsque, pour tout $a>0$, on a

$$
\begin{aligned}
& \sup _{p \in \mathbb{N}} \max \left\{\sup _{P ; p=|P|} \sup _{x \in E} \frac{\left|F^{P}(x)\right|}{p ! M_{a p}^{(\phi)}},\right. \\
& \left.\sup _{J ;|J| \leq p} \sup _{\substack{(\zeta, x) \in E^{2} \\
\zeta \neq x}} \frac{\left|R_{\zeta}^{J, p} F(x)\right|}{j ! M_{a(p+1)}^{(\phi)}|\zeta-x|^{p+1-j}}\right\}<+\infty .
\end{aligned}
$$

On définit ainsi de nouvelles intersections de classes de fonctions et de classes de jets. Toute fonction de $\widehat{\phi}$ définit un jet de $\widehat{J \phi}(E)$ via l'application de restriction

$$
R_{E}: f \mapsto\left(f_{\mid E}^{L}\right)_{L \in \mathbb{N}^{n}}
$$

On dit que $f=F$ au sens des jets sur $E$ lorsque $R_{E}(f)=F$.

On dit que la fonction $\phi$ est non quasi-analytique lorsque la suite $\left(M_{a p}^{(\phi)}\right)_{p \geq 0}$ est non quasi-analytique, pour tout $a>0$. Cela signifie que la 
fonction $\phi$ vérifie la condition suivante :

$$
\forall a>0, \quad \int_{1}^{+\infty} \frac{d t}{t(\exp (a t))^{1 / t}}<+\infty .
$$

Lorsque $\phi$ est la fonction définie par $\phi(x)=x \ln (1+x)$, on retrouve l'intersection des classes de Gevrey : $\widehat{\phi}=\widehat{G}$ et $\widehat{J \phi}(E)=\widehat{G}(E)$. Si $\phi$ vérifie la condition suivante :

$$
\exists B \in \mathbb{R}_{+}, \forall x \in \mathbb{R}_{+}, \quad \phi(2 x) \leq B x+2 \phi(x),
$$

on retrouve le cas d'une intersection à croissance modérée. Les intersections construites ici sont plus riches que les intersections à croissance modérée. En effet, si $f$ est une fonction de classe $C^{\infty}$ sur $\mathbb{R}^{n}$, il existe une fonction $\phi$ non quasi-analytique telle que $R_{E}(f)$ appartienne à $\widehat{J \phi}(E)$.

Dans un premier temps, on démontre un théorème d'extension de type Whitney :

THÉORÈme A. On suppose que la fonction $\phi$ est non quasi-analytique. Soit $F$ un jet appartenant $\grave{a} \widehat{J \phi}(E)$. Il existe une fonction $f$ appartenant $\grave{a}$ $\widehat{\phi}$, à support compact, telle que $f=F$ au sens des jets sur $E$.

Il est ensuite naturel d'étudier si cette extension, de $\widehat{J \phi}(E)$ dans $\widehat{\phi}$, peut être réalisée à l'aide d'un opérateur linéaire continu. On établit :

ThÉorème B. (i) Si $E=\{0\}$ un tel opérateur n'existe pas.

(ii) En dimension 1, si $E=[0,1]$ et si l'intersection est à croissance modérée, un tel opérateur n'existe pas.

En particulier, dans le cas de l'intersection des classes de Gevrey, il n'y a pas d'opérateur d'extension linéaire.

Soit $r$ un réel supérieur ou égal à 2 . On dit que $E$ vérifie la propriété de Markov $\mathbf{M}(r)$ lorsqu'il existe une constante $\mathcal{M}>0$ telle que, pour tout polynôme $Q$ et pour tout multi-indice $J$ de $\mathbb{N}^{n}$, on ait $\left\|D^{J} Q\right\|_{E} \leq$ $\mathcal{M}(\operatorname{deg} Q)^{r|J|}\|Q\|_{E}$. Pour des compacts ayant une telle "régularité" et pour de "grandes classes", en utilisant un procédé d'approximation polynômiale, on montre le théorème suivant :

THÉORÈme C. On suppose que la fonction $\phi$ est non quasi-analytique. Si E est un compact de $\mathbb{R}^{n}$ vérifiant la propriété de Markov $\mathbf{M}(r)$ et si

$$
\lim _{u \rightarrow+\infty} \frac{\phi(u)}{u \ln u}=+\infty
$$

alors il existe un opérateur d'extension linéaire continu de $\widehat{J \phi}(E)$ dans $\widehat{\phi}$.

L'intervalle $[0,1]$ a la propriété de Markov. Pour d'autres exemples de compacts ayant cette propriété, on pourra consulter [13]. 
On peut aussi établir, à l'instar de H. Whitney dans [18] et de M. Valdivia dans [17], des théorèmes d'extension avec réelle analyticité sur $\mathbb{R}^{n} \backslash E$. On a, par exemple :

ThÉORÈme D. On suppose que la fonction $\phi$ est non quasi-analytique. Soit $F \in \widehat{J \phi}(E)$. Il existe une fonction $f \in \widehat{\phi}$, réelle analytique sur $\mathbb{R}^{n} \backslash E$, telle que $f=F$ au sens des jets sur $E$.

Soient $E_{1}$ et $E_{2}$ deux compacts non vides. On dit que $E_{1}$ et $E_{2}$ sont régulièrement situés s'ils sont disjoints ou s'il existe un ouvert borné $\Omega$ de $\mathbb{R}^{n}$, contenant $E_{1} \cup E_{2}$, et deux constantes $\alpha \geq 1$ et $L>0$ tels que

$$
\forall x \in \Omega, \quad d\left(x, E_{1}\right)+d\left(x, E_{2}\right) \geq \operatorname{Ld}\left(x, E_{1} \cap E_{2}\right)^{\alpha} .
$$

On établit un théorème de Łojasiewicz sur la régulière situation.

THÉORÈme E. On suppose que la fonction $\phi$ est non quasi-analytique. Soient $E_{1}$ et $E_{2}$ deux compacts de $\mathbb{R}^{n}$. Alors $E_{1}$ et $E_{2}$ sont régulièrement situés si et seulement si, pour tout couple $\left(F_{1}, F_{2}\right)$ appartenant à $\widehat{J \phi}\left(E_{1}\right) \times$ $\widehat{J \phi}\left(E_{2}\right)$ et vérifiant $F_{1}=F_{2}$ sur $E_{1} \cap E_{2}$ au sens des jets, le jet $F=F_{1} \cup F_{2}$ défini par $F(x)=F_{1}(x)$ si $x \in E_{1}$ et $F(x)=F_{2}(x)$ si $x \in E_{2}$ appartient à $\widehat{J \phi}\left(E_{1} \cup E_{2}\right)$.

Enfin, on peut vérifier que les intersections de classes construites ici héritent des propriétés obtenues par J. Chaumat et A.-M. Chollet dans [3] et [4]. En effet, dans tous leurs travaux, on peut constater qu'il apparaît à chaque fois une perte de régularité "typique" de " $p ! M_{a p}^{(\phi)}$ " vers " $p ! M_{a \lambda(p+\mu)}^{(\phi)}$ " où $\lambda$ et $\mu$ sont deux entiers strictement positifs. Une telle perte est donc masquée par l'intersection. Ainsi les théorèmes de division de Łojasiewicz et de composition de Glaeser s'étendent naturellement aux classes $\widehat{J \phi}(E)$.

La plupart des résultats démontrés dans ce travail ont été annoncés dans une note aux Comptes Rendus de l'Académie des Sciences [C. R. Acad. Sci. Paris 331 (2000), 25-30.]

\section{Définitions et notations}

2.1. Quelques propriétés de suites. Dans tout ce qui suit, toutes les suites $\left(M_{p}\right)_{p \geq 0}$ seront logarithmiquement convexes et vérifieront $M_{0} \leq M_{1}$.

2.1.1. Définitions. On dit que la suite $\left(M_{p}\right)_{p \geq 0}$ est non analytique lorsque

$$
\lim _{p \rightarrow+\infty} M_{p}^{1 / p}=+\infty
$$

On dit que la suite $\left(M_{p}\right)_{p \geq 0}$ est non quasi-analytique lorsque

$$
\sum_{p \geq 1} \frac{M_{p-1}}{p M_{p}}<+\infty .
$$


On sait ([7], chapitre 1) que la condition (2) est équivalente à

$$
\sum_{p \geq 1} \frac{1}{p\left(M_{p}\right)^{1 / p}}<+\infty
$$

et qu'elle implique donc (1).

Soit $\left(M_{p}\right)_{p \geq 0}$ une suite non analytique. On pose $m_{0}=1$ et pour tout $p \in \mathbb{N}^{*}, m_{p}=M_{p} / M_{p-1}$. La suite $\left(m_{p}\right)_{p \geq 0}$ ainsi définie est croissante et vérifie

$$
\lim _{p \rightarrow+\infty} m_{p}=+\infty \quad \text { et } \forall p \in \mathbb{N}, \quad \frac{M_{p}}{M_{0}} \leq\left(m_{p}\right)^{p} .
$$

2.1.2. Fonction $N$ associée à une suite non analytique. Soit $\left(M_{p}\right)_{p \geq 0}$ une suite non analytique. Soit $r>0$. La suite $\left(M_{p+1} r^{p}\right)_{p \geq 0}$ est décroissante puis croissante; on désigne par $N(r)$ le plus petit entier réalisant la borne inférieure de l'ensemble $\left\{M_{p+1} r^{p}: p \geq 0\right\}$. Ainsi $N(r)$ est le plus petit entier $p$ tel que $m_{p+2} \geq 1 / r$ et on a

$$
\begin{aligned}
\forall(j, q) \in \mathbb{N}^{2}, & j \leq q \leq N(r) \Rightarrow r^{q} M_{q+1} \leq r^{j} M_{j+1}, \\
\forall(j, q) \in \mathbb{N}^{2}, & N(r) \leq j \leq q \Rightarrow r^{j} M_{j+1} \leq r^{q} M_{q+1}, \\
\forall j \in \mathbb{N}, & r^{N(r)} M_{N(r)+1} \leq r^{j} M_{j+1} .
\end{aligned}
$$

Enfin, on a également

$$
\lim _{r \rightarrow 0} N(r)=+\infty
$$

2.2. Jets. On note $E$ un compact de $\mathbb{R}^{n}$.

2.2.1. Notations. Soit $J=\left(j_{1}, \ldots, j_{n}\right) \in \mathbb{N}^{n}$ un multi-indice de $\mathbb{N}^{n}$. On note $|J|$ ou $j$ sa longueur, $|J|=j=j_{1}+\ldots+j_{n}$ et $J !=j_{1} ! \ldots j_{n}$ !. Pour tout $x=\left(x_{1}, \ldots, x_{n}\right) \in \mathbb{R}^{n}$, on pose $x^{J}=x_{1}^{j_{1}} \ldots x_{n}^{j_{n}}$ et $|x|=\sqrt{x_{1}^{2}+\ldots+x_{n}^{2}}$.

Soit $f$ une fonction de classe $C^{\infty}$ en $x \in \mathbb{R}^{n}$; pour tout multi-indice $L=\left(l_{1}, \ldots, l_{n}\right) \in \mathbb{N}^{n}$ de longueur $l$ on note

$$
f^{L}(x)=D^{L} f(x)=\frac{\partial^{l} f}{\partial^{l_{1}} x_{1} \ldots \partial^{l_{n}} x_{n}}(x) .
$$

2.2.2. DÉfinitions. Un jet $F$ sur $E$ est la donnée d'une suite $\left(F^{J}\right)_{J \in \mathbb{N}^{n}}$ de fonctions continues sur $E$, à valeurs dans $\mathbb{R}$.

Soient $F$ un jet sur $E$ et $L$ un multi-indice de $\mathbb{N}^{n}$. On définit la $L$-ième dérivée du jet $F$ par $D^{L} F=\left(F^{J+L}\right)_{J \in \mathbb{N}^{n}}$.

Soient $F$ un jet sur $E, p \in \mathbb{N}$ et $\zeta \in E$. On définit le polynôme de Taylor de $F$ d'ordre $p$ par

$$
\forall x \in \mathbb{R}^{n}, \quad T_{\zeta}^{p} F(x)=\sum_{J ;|J| \leq p} \frac{1}{J !}(x-\zeta)^{J} F^{J}(\zeta) .
$$


Avec ces notations, si $p \in \mathbb{N}$ et $J \in \mathbb{N}^{n}$ sont tels que $|J| \leq p$, on a

$$
\forall \zeta \in E, \forall x \in \mathbb{R}^{n}, \quad D^{J}\left(T_{\zeta}^{p} F\right)(x)=T_{\zeta}^{p-j}\left(D^{J} F\right)(x) .
$$

Soient $p \in \mathbb{N}$ et $J \in \mathbb{N}^{n}$ tels que $|J| \leq p,(\zeta, x) \in E^{2}$. On pose

$$
R_{\zeta}^{J, p} F(x)=F^{J}(x)-\sum_{K ;|J+K| \leq p} \frac{1}{K !}(x-\zeta)^{K} F^{J+K}(\zeta) .
$$

On dit que $F$ est un jet de Whitney de classe $C^{\infty}$ sur $E$ lorsque, pour tout $p \in \mathbb{N}$ et tout $J \in \mathbb{N}^{n}$ tels que $|J| \leq p$, on a

$$
R_{\zeta}^{J, p} F(x)=o\left(|x-\zeta|^{p-j}\right),
$$

dès que $|x-\zeta|$ tend vers 0 , avec $(x, \zeta) \in E^{2}$.

2.3. Classes de jets et de fonctions. Soient $E$ un compact de $\mathbb{R}^{n}, \Omega$ un ouvert de $\mathbb{R}^{n}$ et $\left(M_{p}\right)_{p \geq 0}$ une suite non analytique.

2.3.1. Classe $\left\{p ! M_{p}, C_{2}, E\right\}$. Soit $C_{2}>0 ;\left\{p ! M_{p}, C_{2}, E\right\}$ est l'ensemble des jets $F$, définis sur $E$, pour lesquels il existe $C_{1} \geq 0$ tel que

$$
\sup _{p \in \mathbb{N}} \sup _{P ; p=|P|} \sup _{x \in E} \frac{\left|F^{P}(x)\right|}{C_{2}^{p} p ! M_{p}} \leq C_{1}
$$

et, pour tout entier $p$ et pour tout multi-indice $J$ de longueur $j \leq p$,

$$
\forall(x, \zeta) \in E^{2},\left|R_{\zeta}^{J, p} F(x)\right| \leq C_{1} C_{2}^{p+1} j ! M_{p+1}|\zeta-x|^{p+1-j} .
$$

En prenant pour norme du jet $\|F\|_{\left\{p ! M_{p}, C_{2}, E\right\}}$ la plus petite constante $C_{1}$ réalisant (6) et (7), $\left\{p ! M_{p}, C_{2}, E\right\}$ est un espace de Banach.

2.3.2. Classe $\left\{p ! M_{p}, C_{2}, \Omega\right\}$. Soit $C_{2}>0 ;\left\{p ! M_{p}, C_{2}, \Omega\right\}$ désigne l'ensemble des fonctions de classe $C^{\infty}$ sur $\Omega$ telles que

$$
\sup _{p \in \mathbb{N}} \sup _{P ; p=|P|} \sup _{x \in \Omega} \frac{\left|f^{P}(x)\right|}{C_{2}^{p} p ! M_{p}}=\|f\|_{\left\{p ! M_{p}, C_{2}, \Omega\right\}}<+\infty .
$$

Muni de cette norme, $\left\{p ! M_{p}, C_{2}, \Omega\right\}$ est un espace de Banach.

2.3.3. Application de restriction. Soit $E$ un compact de $\mathbb{R}^{n}$. Toute fonction de $\left\{p ! M_{p}, C_{2}, \mathbb{R}^{n}\right\}$ définit un jet de $\left\{p ! M_{p}, 2 n C_{2}, E\right\}$ via l'application de restriction

$$
R_{E}:\left\{p ! M_{p}, C_{2}, \mathbb{R}^{n}\right\} \rightarrow\left\{p ! M_{p}, 2 n C_{2}, E\right\}, \quad f \mapsto\left(\left.D^{L} f\right|_{E}\right)_{L \in \mathbb{N}^{n}} .
$$

De plus $\left\|R_{E}(f)\right\|_{\left\{p ! M_{p}, 2 n C_{2}, E\right\}} \leq\|f\|_{\left\{p ! M_{p}, C_{2}, \mathbb{R}^{n}\right\}}$.

2.3.4. Suite associée à une fonction. Dans tout ce travail, $\phi$ désigne une fonction convexe et croissante sur $\mathbb{R}_{+}$vérifiant $\lim _{t \rightarrow+\infty} \phi(t) / t=+\infty$. 
Pour tout $a>0$, on définit la suite logarithmiquement convexe $\left(M_{a p}^{(\phi)}\right)_{p \geq 0}$ en posant

$$
\forall p \in \mathbb{N}, \quad M_{a p}^{(\phi)}=\exp (\phi(a p)) .
$$

La suite $\left(M_{a p}^{(\phi)}\right)_{p \geq 0}$ est non analytique, pour tout $a>0$.

Soit $a>0$. La suite $\left(M_{a p}^{(\phi)}\right)_{p \geq 0}$ est non quasi-analytique si et seulement si

$$
\int_{1}^{+\infty} \frac{d t}{t(\exp \phi(a t))^{1 / t}}<+\infty .
$$

Si, pour tout $a>0$, la suite $\left(M_{a p}^{(\phi)}\right)_{p \geq 0}$ est non quasi-analytique, on dit que $\phi$ est non quasi-analytique.

\subsubsection{Intersections de classes}

DÉFinitions. On définit les intersections de classes suivantes :

$$
\widehat{J \phi}(E)=\bigcap_{a>0}\left\{p ! M_{a p}^{(\phi)}, 1, E\right\} \quad \text { et } \quad \widehat{\phi}(\Omega)=\bigcap_{a>0}\left\{p ! M_{a p}^{(\phi)}, 1, \Omega\right\} .
$$

Muni de la famille de normes $\|\cdot\|_{\left\{p ! M_{a p}^{(\phi)}, 1, E\right\}}\left(\right.$ resp. $\left.\|\cdot\|_{\left\{p ! M_{a p}^{(\phi)}, 1, \Omega\right\}}\right), \widehat{J \phi}(E)$ (resp. $\widehat{\phi}(\Omega)$ ) est un espace de Fréchet.

Lorsqu'il n'y aura pas d'ambiguïté, on notera $\widehat{\phi}$ à la place de $\widehat{\phi}\left(\mathbb{R}^{n}\right)$. Soit $E$ un compact non vide de $\mathbb{R}^{n}$; alors, comme au 2.3.3, toute fonction de $\widehat{\phi}\left(\mathbb{R}^{n}\right)$ définit un jet de $\widehat{J \phi}(E)$ via l'application de restriction

$$
R_{E}: \widehat{\phi}\left(\mathbb{R}^{n}\right) \rightarrow \widehat{J \phi}(E), \quad f \mapsto\left(\left.D^{L} f\right|_{E}\right)_{L \in \mathbb{N}^{n}} .
$$

De plus $R_{E}$ est une application linéaire continue.

3. Extension avec perte au-dessus d'un compact. Le résultat principal de ce paragraphe est constitué par le théorème 3.1. C'est un théorème d'extension de type Whitney, avec perte de régularité, dont la preuve s'inspire de [1] et [2]. On utilise un développement de Taylor d'ordre variable que l'on régularise à l'aide d'une partition de l'unité.

3.1. ThÉorème. Soient $\left(M_{p}\right)_{p \geq 0}$ une suite non quasi-analytique, $E$ un compact de $\mathbb{R}^{n}$ et $C_{2}>0$. Il existe une constante $U_{2}>0$ et une application linéaire continue $T$ de $\left\{p ! M_{p}, C_{2}, E\right\}$ dans $\left\{p ! M_{p} M_{p+1}, C_{2} U_{2}, \mathbb{R}^{n}\right\}$ telle que, pour tout jet $F$ appartenant $\grave{a}\left\{p ! M_{p}, C_{2}, E\right\}$, on a $T(F)=F$ au sens des jets sur $E$. De plus, la constante $U_{2}$ ne dépend que de $E$ et de la suite $\left(M_{p}\right)_{p \geq 0}$.

On rappelle que $T(F)=F$ au sens des jets sur $E$ signifie que, pour tout multi-indice $J$ et tout $x \in E$, on a

$$
F^{J}(x)=(T(F))^{J}(x)
$$


Le lemme suivant est classique ([2], [7]).

3.1.1. Lemme. Soient $\left(M_{p}\right)_{p \geq 0}$ une suite non quasi-analytique et $\delta \geq$ $\sum_{p=1}^{+\infty} 1 /\left(p_{p}\right)$. Pour tout $t>1$, il existe une fonction $q_{t}$ de classe $C^{\infty}$ sur $\mathbb{R}$ et à support dans $[-t, t]$ telle que

$$
\forall x \in[-1,1], \quad q_{t}(x)=1
$$

et

$$
\forall j \in \mathbb{N}, \forall x \in \mathbb{R}, \quad\left|q_{t}^{(j)}(x)\right| \leq 2^{j}\left(\frac{\delta}{t-1}\right)^{j} j ! \frac{M_{j}}{M_{0}} .
$$

3.1.2. Lemme de ReCouvrement De $\mathbb{R}^{n} \backslash E$ [5]. Soit $E$ un compact de $\mathbb{R}^{n}$; on désigne par $\varrho(x)$ la distance de $x$ à $E$. Soit $t^{\prime}>1$. Il existe des constantes $a, b \in \mathbb{R}, n_{0} \in \mathbb{N}$ telles que $0<a<1<b, n_{0}>1$ et une famille dénombrable de boules $B\left(x_{i}, r_{i}\right), i \in \mathbb{N}$, vérifiant:

(a) $\mathbb{R}^{n} \backslash E=\bigcup_{i \in \mathbb{N}} B\left(x_{i}, r_{i}\right)=\bigcup_{i \in \mathbb{N}} B\left(x_{i}, t^{\prime} r_{i}\right)$,

(b) $x \in B\left(x_{i}, t^{\prime} r_{i}\right) \Rightarrow a r_{i} \leq \varrho(x) \leq b r_{i}$ et $a \varrho(x) \leq \varrho\left(x_{i}\right) \leq b \varrho(x)$,

(c) tout $x \in \mathbb{R}^{n} \backslash E$ appartient au plus à $n_{0}$ boules $B\left(x_{i}, t^{\prime} r_{i}\right)$.

3.1.3. Partition de l'unité associée. Soit $t>1$. Pour $y=\left(y_{1}, \ldots, y_{n}\right)$ $\in \mathbb{R}^{n}$ on pose

$$
Q_{t}(y)=q_{t}\left(y_{1}\right) \ldots q_{t}\left(y_{n}\right) .
$$

Soit $\left\{B\left(x_{i}, r_{i}\right): i \in \mathbb{N}\right\}$ la famille de boules donnée par le lemme précédent lorsque $t^{\prime}=\sqrt{n} t$. Pour tout $i \geq 1$, on pose $\psi_{i}(x)=Q_{t}\left(\left(x-x_{i}\right) / r_{i}\right)$, puis

$$
\phi_{0}=\psi_{0}
$$

et, pour tout $i \geq 1$,

$$
\phi_{i}=\psi_{i}\left(1-\psi_{0}\right)\left(1-\psi_{1}\right) \ldots\left(1-\psi_{i-1}\right) .
$$

Alors $\left(\phi_{i}\right)_{i \in \mathbb{N}}$ est une partition de l'unité associée au recouvrement $\bigcup_{i \in \mathbb{N}} B\left(x_{i}, t^{\prime} r_{i}\right)$. Il existe une constante $C \geq 1$, ne dépendant que de la suite $\left(M_{p}\right)_{p \geq 0}$, de la dimension $n$, de $t$ et de $E$, telle que, pour tout multi-indice $J$, on ait

$$
\forall x \in \mathbb{R}^{n} \backslash E, \quad\left|D^{J} \phi_{i}(x)\right| \leq\left(\frac{C}{\varrho(x)}\right)^{j} j ! \frac{M_{j}}{M_{0}} .
$$

Dans tout ce qui suit, si $x \in \mathbb{R}^{n} \backslash E$, alors $\widehat{x}$ désigne un point de $E$ réalisant la distance $\varrho(x)$.

3.2. Proposition [2]. Soient $\left(M_{p}\right)_{p \in \mathbb{N}}$ une suite non analytique et $E$ un compact de $\mathbb{R}^{n}$. Si $F$ est un jet appartenant à $\left\{p ! M_{p}, C_{2}, E\right\}$ et si $\|F\|_{\left\{p ! M_{p}, C_{2}, E\right\}}$ désigne la norme introduite au paragraphe 2.3 .1 , alors, pour tout entier $p$, pour tout multi-indice $J$ vérifiant $|J| \leq p$ et pour tout $\left(x, \zeta_{1}, \zeta_{2}\right)$ $\in \mathbb{R}^{n} \times E^{2}$, on a

$$
\left|D^{J}\left(T_{\zeta_{1}}^{p} F-T_{\zeta_{2}}^{p} F\right)(x)\right|
$$




$$
\leq\|F\|_{\left\{p ! M_{p}, C_{2}, E\right\}}\left(2 C_{2} n^{2}\right)^{p+1} j ! M_{p+1}\left(\left|x-\zeta_{1}\right|+\left|\zeta_{1}-\zeta_{2}\right|\right)^{p+1-j} .
$$

Comme dans [2], proposition 9, on a la proposition suivante :

3.3. Proposition. Soient $\left(M_{p}\right)_{p \geq 0}$ une suite non analytique, $E$ un compact de $\mathbb{R}^{n}$ et $F$ un jet appartenant à $\left\{p ! M_{p}, C_{2}, E\right\}$. Si $\alpha \geq 4 n^{2} C_{2}$, alors, pour tout $x \in \mathbb{R}^{n} \backslash E$ et pour tout multi-indice $J$, on a

$$
\left|D^{J}\left(T_{\widehat{x}}^{N(\alpha \varrho(x))} F\right)(x)\right| \leq 2 j !\|F\|_{\left\{p ! M_{p}, C_{2}, E\right\}} M_{j}\left(\frac{\alpha}{n}\right)^{j}
$$

et

$$
\left|D^{J}\left(T_{\widehat{x}}^{N(\alpha \varrho(x))} F\right)(x)-F^{J}(\widehat{x})\right| \leq j !\|F\|_{\left\{p ! M_{p}, C_{2}, E\right\}} M_{j+1} \alpha\left(\frac{\alpha}{n}\right)^{j} \varrho(x) .
$$

Remarques. (i) $D^{J}\left(T_{\widehat{x}}^{N(\alpha \varrho(x))} F\right)(x)$ signifie que l'on a calculé la $J$-ième dérivée du polynôme $T_{\widehat{x}}^{N(\alpha \varrho(x))} F(t)$ puis que l'on a évalué cette dérivée au point $t=x$.

(ii) On rappelle que la fonction $N$ a été définie au 2.1.2.

Démonstration du théorème 3.1. On fixe $t>1$; soient $t^{\prime}=\sqrt{n} t$ et $\left(\phi_{i}\right)_{i \in \mathbb{N}}$ la partition de l'unité obtenue en 3.1.3. Soit $\alpha=2 C_{2} n^{2}\left(2+2 t^{\prime} / a^{2}+\right.$ $1 / a)$. Posons $T(F)=f$ où $f$ est la fonction définie par

$$
\forall x \in \mathbb{R}^{n} \backslash E, \quad f(x)=\sum_{i=0}^{+\infty} \phi_{i}(x) T_{\widehat{x}_{i}}^{N\left(\alpha \varrho\left(x_{i}\right)\right)} F(x)
$$

et

$$
\forall x \in E, \quad f(x)=F^{0}(x) .
$$

$f$ est clairement de classe $C^{\infty}$ sur $\mathbb{R}^{n} \backslash E$ et sur $\stackrel{\circ}{E}$, l'intérieur de $E$. Soient $x \in \mathbb{R}^{n} \backslash E$ et $Q$ un multi-indice; évaluons $D^{Q}\left(f-T_{\widehat{x}}^{N(\alpha \varrho(x))} F\right)(x)$. On peut supposer que $\varrho(x) \leq 1$.

Soient $i \in \mathbb{N}$ et $J$ un multi-indice tel que $J \leq Q$. On pose

$$
\begin{aligned}
& E_{1}=D^{J}\left(T_{\widehat{x}_{i}}^{N\left(\alpha \varrho\left(x_{i}\right)\right)} F-T_{\widehat{x}}^{N\left(\alpha \varrho\left(x_{i}\right)\right)} F\right)(x), \\
& E_{2}=D^{J}\left(T_{\widehat{x}}^{N\left(\alpha \varrho\left(x_{i}\right)\right)} F-T_{\widehat{x}}^{N(\alpha \varrho(x))} F\right)(x) .
\end{aligned}
$$

Comme dans la preuve du théorème 11 de [2], on a

$$
\left|E_{1}\right| \leq j !\|F\|(b \alpha)^{q+1} M_{q+1} \varrho(x)^{q+1-j}
$$

et

$$
\left|E_{2}\right| \leq 4 C_{2} n^{2} j !\|F\|(b \alpha)^{q} M_{q+1}(\varrho(x))^{q+1-j} .
$$

En utilisant la formule de Leibniz, on obtient

$$
\left|D^{Q}\left(f-T_{\widehat{x}}^{N((\alpha \varrho(x))} F(x)\right)(x)\right| \leq q ! C_{3}\|F\| C_{4}^{q} M_{q} M_{q+1} \varrho(x)
$$


où $C_{3}=2 n_{0} b \alpha / M_{0}$ et $C_{4}=2 n^{2} b \alpha C ; C$ désigne ici la constante de la partition de l'unité 3.1.3.

Puisque $\varrho(x) \leq 1$, on en déduit que

$$
\left|D^{Q}(f)(x)\right| \leq U_{1}\|F\|\left(C_{2} U_{2}\right)^{q} q ! M_{q} M_{q+1}
$$

où $U_{1}=\left(2 n_{0} b \alpha+2\right) / M_{0}$ et $U_{2}=4 n^{4} b C\left(2+2 t^{\prime} / a^{2}+1 / a\right)$. La constante $U_{1}$ ne dépend que de $E$, de la suite $\left(M_{p}\right)_{p \geq 0}$ et de $C_{2}$. La constante $U_{2}$ ne dépend que de $E$ et de la suite $\left(M_{p}\right)_{p \geq 0}$.

Soit $x_{0} \in E \backslash \stackrel{\circ}{E}$; on déduit de (10) et de (13) que l'on a, pour tout multi-indice $Q$,

$$
\lim _{\substack{x \rightarrow x_{0} \\ x \in \mathbb{R}^{n} \backslash E}} D^{Q}(f)(x)=F^{Q}\left(x_{0}\right) .
$$

D'après le lemme d'Hesténes ([16], page 80), $f$ est de classe $C^{\infty}$ sur $\mathbb{R}^{n}$. De plus, compte tenu de (14), $f \in\left\{p ! M_{p} M_{p+1}, C_{2} \times U_{2}, \mathbb{R}^{n}\right\}$, ce qui démontre le théorème 3.1.

REmarque. En multipliant par une fonction troncature, on peut obtenir une fonction à support compact.

\section{Extensions de jets dans des intersections de classes}

4.1. ThÉORÈme D'EXTEnsion DE $\widehat{J \phi}(E)$ DANS $\widehat{\phi}$. Soit $\phi$ une fonction non quasi-analytique et soit $F$ un jet appartenant à $\widehat{J \phi}(E)$. Il existe une fonction $f \in \widehat{\phi}$, à support compact, telle que l'on ait $f=F$ sur $E$ au sens des jets.

On rappelle qu'une fonction $\phi$ non quasi-analytique est une fonction convexe sur $\mathbb{R}_{+}$, croissante et vérifiant la condition suivante :

$$
\forall a>0, \quad \int_{1}^{+\infty} \frac{d t}{t(\exp \phi(a t))^{1 / t}}<+\infty .
$$

On établit d'abord l'analogue de la proposition clé de [3].

4.2. Proposition. Soit $\phi$ une fonction non quasi-analytique. Soit $\left(u_{p}\right)_{p \geq 0}$ une suite vérifiant

$$
\forall a>0, \exists C_{1}(a) \geq 0, \forall p \in \mathbb{N}, \quad\left|u_{p}\right| \leq C_{1}(a) M_{a p}^{(\phi)} .
$$

Alors il existe une fonction $\psi$ convexe sur $\mathbb{R}_{+}$, croissante et vérifiant

$$
\begin{gathered}
\psi(0)=\phi(0), \\
\int_{1}^{+\infty} \frac{d t}{t(\exp \psi(t))^{1 / t}}<+\infty, \\
\forall a>0, \exists C_{2}(a)>0, \forall p \in \mathbb{N}, \quad M_{p}^{(\psi)} \leq C_{2}(a) M_{a p}^{(\phi)},
\end{gathered}
$$




$$
\exists A>0, \forall p \in \mathbb{N}, \quad\left|u_{p}\right| \leq A M_{p}^{(\psi)} .
$$

Remarque. Notons tout d'abord que sous l'hypothèse (15), la condition (18) est équivalente à

$$
\begin{aligned}
\forall a>0, \exists C_{2}(a)>0, \exists C_{3}(a)>0, \forall p & \in \mathbb{N}, \\
& M_{p}^{(\psi)} \leq C_{2}(a)\left(C_{3}(a)\right)^{p} M_{a p}^{(\phi)} .
\end{aligned}
$$

Bien sûr (18) implique (20). Réciproquement, soit $a>0$ et soient $C_{2}(a / 2)$ et $C_{3}(a / 2)$ les constantes données par (20). (15) implique $\lim _{t \rightarrow+\infty}\left(M_{a t / 2}^{(\phi)}\right)^{1 / t}=$ $+\infty$. Donc

$$
\exists t_{0} \in \mathbb{R}_{+}, \forall t \geq t_{0}, \quad\left(M_{a t / 2}^{(\phi)}\right)^{1 / t} \geq C_{3}(a / 2) .
$$

Alors,

$$
\forall t \geq t_{0}, \quad M_{a t / 2}^{(\phi)} \geq\left(C_{3}(a / 2)\right)^{t} .
$$

Donc, quitte à augmenter $C_{2}(a / 2)$, pour tout $p \in \mathbb{N}$, on a

$$
M_{p}^{(\psi)} \leq C_{2}(a / 2)\left(M_{a p / 2}^{(\phi)}\right)^{2} \leq C_{2}(a / 2) M_{0}^{(\phi)} M_{a p}^{(\phi)},
$$

ce qui démontre (18).

Remarque. Dans la démonstration qui suit on note $\phi^{\prime}$ la dérivée à droite de $\phi$.

Démonstration de la proposition. Soit $\left(t_{k}\right)_{k \geq 0}$ une suite de réels strictement croissante vérifiant les conditions suivantes :

$$
\begin{gathered}
t_{0}=0, \quad t_{1}>1, \quad \phi\left(\frac{t_{1}}{2}\right) \geq 0 \quad \text { et } \quad \phi^{\prime}\left(t_{1}\right)>0, \\
\forall k \in \mathbb{N}^{*}, \quad \frac{1}{2} t_{k} \geq t_{k-1}, \\
\forall k \in \mathbb{N}^{*}, \quad \int_{t_{k}}^{+\infty} \frac{d t}{t\left(\exp \phi\left(2^{-k} t\right)\right)^{1 / t}} \leq 2^{-k} .
\end{gathered}
$$

Il est facile de construire une telle suite.

Définissons $\psi$ successivement sur les intervalles $\left[t_{k}, t_{k+1}[\right.$. On définit, par récurrence, une suite de fonctions $\left(\phi_{k}\right)_{k \geq 0}$ en posant $\phi_{0}(t)=\phi(t)$ pour tout $t \in[0,+\infty[$, puis, pour tout entier $k$ et pour tout $t \in[0,+\infty[$,

$$
\begin{aligned}
\phi_{k+1}(t)= & \phi_{k}\left(\frac{1}{2} t\right)+\left(\phi_{k}^{\prime}\left(t_{k+1}\right)-\frac{1}{2} \phi_{k}^{\prime}\left(\frac{1}{2} t_{k+1}\right)\right)\left(t-t_{k+1}\right) \\
& +\phi_{k}\left(t_{k+1}\right)-\phi_{k}\left(\frac{1}{2} t_{k+1}\right)
\end{aligned}
$$

$\phi_{k}^{\prime}$ étant la dérivée à droite de $\phi_{k}$.

PROPRIÉtÉs. (i) $\phi_{k+1}\left(t_{k+1}\right)=\phi_{k}\left(t_{k+1}\right)$ et $\phi_{k+1}^{\prime}\left(t_{k+1}\right)=\phi_{k}^{\prime}\left(t_{k+1}\right)$, pour tout entier $k$. 
(ii) Il existe deux suites de réels, $\left(A_{k}\right)_{k \geq 0}$ et $\left(B_{k}\right)_{k \geq 0}$, telles que, pour tout entier $k$, on ait

$$
\forall t \in\left[0,+\infty\left[, \quad \phi_{k}(t)=\phi\left(2^{-k} t\right)+A_{k} t+B_{k} .\right.\right.
$$

De plus, $A_{k}>0$ pour tout entier $k>0$.

(iii) Pour tout entier $k$ et pour tout $t \in\left[t_{k},+\infty\left[\right.\right.$, on a $A_{k} t+B_{k} \geq 0$.

(iv) Pour tout entier $k$ et pour tout $t \in\left[t_{k+1},+\infty\left[\right.\right.$, on a $\phi_{k+1}(t) \leq$ $\phi\left(2^{-(k+1)} t\right)+\phi_{k}(t)$.

Les points (i), (ii) et (iii) sont clairs. Pour (iv) remarquons que (21) et (22) impliquent $\phi\left(2^{-(k+1)} t_{k+1}\right) \geq 0$. Ainsi, en utilisant (ii), pour tout $t \in\left[t_{k+1},+\infty[\right.$, on a $\phi_{k+1}(t) \leq \phi\left(2^{-(k+1)} t\right)+A_{k} t+B_{k}+2^{-k} \phi^{\prime}\left(2^{-k} t_{k+1}\right)\left(t-t_{k+1}\right)+\phi\left(2^{-k} t_{k+1}\right)$. Or, $y=2^{-k} \phi^{\prime}\left(2^{-k} t_{k+1}\right)\left(t-t_{k+1}\right)+\phi\left(2^{-k} t_{k+1}\right)$ est l'équation de la tangente en $t_{k+1}$ de la fonction convexe $t \mapsto \phi\left(2^{-k} t\right)$. On a donc $\phi_{k+1}(t) \leq \phi\left(2^{-(k+1)} t\right)+$ $A_{k} t+B_{k}+\phi\left(2^{-k} t\right)=\phi\left(2^{-(k+1)} t\right)+\phi_{k}(t)$ pour tout $t \in\left[t_{k+1},+\infty[\right.$.

Assertion. Quitte à augmenter les termes de la suite $\left(t_{k}\right)_{k \geq 0}$ en préservant la condition (22), on a

$$
\forall k \in \mathbb{N}^{*}, \forall j \in\{0, \ldots, k-1\}, \forall t \in\left[t_{k+1},+\infty\left[, \quad \phi_{k+1} \leq \phi_{j} .\right.\right.
$$

D'après la propriété (iv) précédente, on a

$$
\forall t \in\left[t_{k+1},+\infty\left[, \quad \phi_{k+1}(t) \leq \phi\left(2^{-(k+1)} t\right)+\phi_{k}(t)\right.\right.
$$

et, d'autre part,

$$
\forall m \in \mathbb{N}, \quad \phi_{m}(t) \underset{t \rightarrow+\infty}{\sim} \phi\left(2^{-m} t\right) .
$$

Soit $\varepsilon$ un réel strictement positif. Il existe $T_{k+1}>t_{k}$ tel que

$$
\begin{aligned}
\forall j \in\{0, \ldots, k\}, \quad \forall t \in\left[T_{k+1},+\infty[,\right. \\
\\
\phi\left(2^{-j} t\right) \leq(1+\varepsilon) \phi_{j}(t) \quad \text { et } \quad \phi_{k}(t) \leq(1+\varepsilon) \phi\left(2^{-k} t\right) .
\end{aligned}
$$

De plus, par convexité de la fonction $\phi$, on a

$$
\phi\left(2^{-k} t\right) \leq 2^{-k+j} \phi\left(2^{-j} t\right)+\left(1-2^{-k+j}\right) \phi(0) \leq \frac{1}{2} \phi\left(2^{-j} t\right)+\left(1-2^{-k+j}\right) \phi(0) .
$$

Comme $\lim _{t \rightarrow+\infty} \phi\left(2^{-j} t\right)=+\infty$, il existe $T_{k+1}^{\prime} \geq T_{k+1}$ tel que

$$
\forall j \in\{0, \ldots, k-1\}, \forall t \in\left[T_{k+1}^{\prime},+\infty\left[, \quad\left(1-2^{-k+j}\right) \phi(0) \leq \varepsilon \phi\left(2^{-j} t\right) .\right.\right.
$$

On a alors

$$
\forall j \in\{0, \ldots, k-1\}, \forall t \in\left[T_{k+1}^{\prime},+\infty\left[, \quad \phi_{k}(t) \leq(1+\varepsilon)^{2}\left(\frac{1}{2}+\varepsilon\right) \phi_{j}(t) .\right.\right.
$$

De même

$$
\begin{aligned}
\phi\left(2^{-(k+1)} t\right) & \leq 2^{-k+1+j} \phi\left(2^{-j} t\right)+\left(1-2^{-k+1+j}\right) \phi(0) \\
& \leq \frac{1}{4} \phi\left(2^{-j} t\right)+\left(1-2^{-k+1+j}\right) \phi(0)
\end{aligned}
$$


et il existe $T_{k+1}^{\prime \prime} \geq T_{k+1}^{\prime}$ tel que

$\forall j \in\{0, \ldots, k-1\}, \forall t \in\left[T_{k+1}^{\prime \prime},+\infty\left[, \quad \phi\left(2^{-(k+1)} t\right) \leq\left(\frac{1}{4}+\varepsilon\right)(1+\varepsilon) \phi_{j}(t)\right.\right.$.

En choisissant $t_{k+1} \geq T_{k+1}^{\prime \prime}$, tout en préservant la condition (22), pour tout $j \in\{0, \ldots, k-1\}$ et tout $t \in\left[t_{k+1},+\infty[\right.$, on a

$$
\phi_{k+1}(t) \leq\left[\frac{3}{4}+\frac{13}{4} \varepsilon+\frac{7}{2} \varepsilon^{2}+\varepsilon^{3}\right] \phi_{j}(t) .
$$

L'assertion est démontrée en choisissant, par exemple, $\varepsilon=1 / 100$.

Définition de $\psi$. Soit $\psi$ la fonction définie sur $\mathbb{R}_{+}$par

$$
\forall k \in \mathbb{N}, \forall t \in\left[t_{k}, t_{k+1}\left[, \quad \psi(t)=\phi_{k}(t) .\right.\right.
$$

La fonction $\psi$ est convexe, croissante, de limite $+\infty$ en $+\infty$ et $\psi(0)=\phi(0)$. Montrons que $\psi$ vérifie (20), (17), (19).

Notons, pour tout $p \in \mathbb{N}$,

$$
M_{p}^{(\psi)}=\exp \psi(p)=C_{k}^{p} C_{k}^{\prime} M_{2^{-k} p}^{(\phi)}
$$

où $k$ est l'entier vérifiant $p \in\left[t_{k}, t_{k+1}\left[, C_{k}=\exp A_{k}\right.\right.$ et $C_{k}^{\prime}=\exp B_{k}$.

Soit $a>0$; il existe $k_{0} \in \mathbb{N}$ tel que $2^{-k_{0}}<a$. Si $k \geq k_{0}+2$ et $p \in$ $\left[t_{k}, t_{k+1}\left[\right.\right.$, alors $M_{p}^{(\psi)}=\exp \left(\phi_{k}(p)-\phi(a p)\right) M_{a p}^{(\phi)}$ et, par (25) et (iv), on a $\phi_{k}(p) \leq \phi(a p)+A_{k_{0}} p+B_{k_{0}}$. Donc $M_{p}^{(\psi)} \leq C_{k_{0}}^{p} C_{k_{0}}^{\prime} M_{a p}^{(\phi)}$ pour tout $p \geq t_{k_{0}+2}$. Comme il n'y a qu'un nombre fini d'entiers $p<t_{k_{0+2}}$, on en déduit (20).

Pour tout $k \in \mathbb{N}^{*}$ et tout $t \in\left[t_{k}, t_{k+1}\left[\right.\right.$, on a $\psi(t)=\phi_{k}(t) \geq \phi\left(2^{-k} t\right)$. En utilisant (23), on a

$$
\sum_{k=1}^{+\infty} \int_{t_{k}}^{t_{k+1}} \frac{d t}{t(\exp \psi(t))^{1 / t}}+\int_{1}^{t_{1}} \frac{d t}{t(\exp \psi(t))^{1 / t}}<+\infty .
$$

Enfin, quitte à augmenter les termes de la suite $\left(t_{k}\right)_{k \geq 0}$ en préservant la condition (22), $\psi$ vérifie la condition (19). En effet, par hypothèse,

$$
\forall a>0, \exists C_{1}(a)>0, \forall p \in \mathbb{N}, \quad\left|u_{p}\right| \leq C_{1}(a) M_{a p}^{(\phi)} .
$$

D'autre part, si $k \in \mathbb{N}^{*}$ et $p \in\left[t_{k}, t_{k+1}\right.$ [, alors

$$
M_{p}^{(\psi)}=C_{k}^{p} C_{k}^{\prime} M_{2^{-k} p}^{(\phi)} \geq \frac{C_{k}^{p} C_{k}^{\prime}\left|u_{p}\right|}{C_{1}\left(2^{-k}\right)}
$$

donc

$$
\left|u_{p}\right| \leq\left(\frac{C_{1}\left(2^{-k}\right)}{C_{k}^{p} C_{k}^{\prime}}\right) M_{p}^{(\psi)}
$$

Quitte à augmenter les termes de la suite $\left(t_{k}\right)_{k \geq 0}$, on peut imposer la condition suivante :

$$
\forall p \in\left[t_{k},+\infty\left[, \quad \frac{C_{1}\left(2^{-k}\right)}{C_{k}^{p} C_{k}^{\prime}} \leq 1,\right.\right.
$$

ce qui achève la preuve de la proposition 4.2. 
Démonstration du théorème 4.1. Soit $F$ un jet appartenant à $\widehat{J \phi}(E)$. D'après la proposition 4.2, il existe une suite $\left(M_{p}^{\prime}\right)_{p \geq 0}=\left(M_{p}^{(\psi)}\right)_{p \geq 0}$ telle que $F \in\left\{p ! M_{p}^{\prime}, 1, E\right\}$. D'après le théorème 3.1, il existe une fonction $f \in$ $\left\{p ! M_{p}^{\prime} M_{p+1}^{\prime}, U_{2}, \mathbb{R}^{n}\right\}$ réalisant l'extension du jet $F$. Cette fonction appartient à $\widehat{\phi}$. En effet, pour tout $a>0$, avec les notations de l'inégalité (18), on a $\forall p \geq 1, \quad M_{p}^{\prime} M_{p+1}^{\prime} \leq\left(M_{p+1}^{\prime}\right)^{2} \leq M_{0}^{\prime} M_{2(p+1)}^{\prime} \leq M_{0}^{\prime} M_{4 p}^{\prime} \leq C_{2}(a / 4) M_{0}^{\prime} M_{a p}$. En utilisant le même argument que pour la démonstration de l'équivalence entre (18) et (20), on établit que $f$ appartient à $\widehat{\phi}=\bigcap_{a>0}\left\{p ! M_{a p}^{(\phi)}, 1, \mathbb{R}^{n}\right\}$.

5. Conditions nécessaires d'extension. On rappelle que dans tout ce travail, $\phi$ désigne une fonction convexe et croissante sur $\mathbb{R}_{+}$vérifiant $\lim _{t \rightarrow+\infty} \phi(t) / t=+\infty$.

5.1. DÉfinition. Soit $\Omega$ un ouvert non vide de $\mathbb{R}^{n}$. On note

$$
\widehat{\phi}_{\text {loc }}(\Omega)=\left\{f \in C^{\infty}(\Omega): \forall K \subset \subset \Omega, R_{K}(f) \in \widehat{J \phi}(K)\right\} .
$$

Comme au 2.3.3, on a noté $R_{K}(f)=\left(\left.D^{L} f\right|_{K}\right)_{L \in \mathbb{N}^{n}} . K \subset \subset \Omega$ signifie que $K$ est un compact de $\Omega$. Lorsque $\Omega$ est un ouvert contenant le compact $E$, le théorème 4.1 reste valide, en remplaçant $\widehat{\phi}$ par $\widehat{\phi}_{\text {loc }}(\Omega)$.

5.2. PropriÉté. $\widehat{\phi}_{\mathrm{loc}}(\Omega)$ est un espace de Fréchet.

Plus précisément, soit $\left(X_{i}\right)_{i \geq 1}$ une suite d'ouverts relativement compacts non vides tels que $\bigcup_{i \geq 1} X_{i}=\Omega$ et $\bar{X}_{i} \subset X_{i+1}$ pour tout entier $i \geq 1$. Alors $\widehat{\phi}_{\text {loc }}(\Omega)$ est un espace de Fréchet dont la topologie est définie par la famille de semi-normes $p_{i}(f)=\left\|R_{\bar{X}_{i}}(f)\right\|_{\left\{p ! M_{(1 / i) p}^{(\phi)}, 1, \bar{X}_{i}\right\}}$.

5.3. Proposition. Soit $E$ un compact non vide de $\mathbb{R}^{n}$. S'il existe un ouvert $\Omega$ contenant $E$ tel que l'opérateur de restriction $R_{E}: \widehat{\phi}_{\mathrm{loc}}(\Omega) \rightarrow$ $\widehat{J \phi}(E)$ soit surjectif, alors $\phi$ est non quasi-analytique.

Démonstration. Soit $\left(x_{0}, y_{0}\right) \in E^{2}$ tel que $\left|x_{0}-y_{0}\right|=\operatorname{diam}(E)$. On peut supposer que $x_{0}$ a pour coordonnées $(-\beta, 0, \ldots, 0)$ et que $y_{0}$ a pour coordonnées $(\beta, 0, \ldots, 0), \beta \geq 0$. Notons $x=\left(x^{\prime}, x^{\prime \prime}\right) \in \mathbb{R} \times \mathbb{R}^{n-1}$. L'application de restriction $R_{E}$ est une application linéaire surjective et continue entre espaces de Fréchet; elle est donc ouverte. Donc, pour tout $i \in \mathbb{N}^{*}$, il existe deux réels $a^{\prime}>0$ et $\varepsilon>0$ tels que

$$
B_{a^{\prime}}(0,2 \varepsilon) \subset R_{E}\left(B_{i}(0,1)\right) .
$$

$B_{i}(0,1)$ désigne la boule unité de $\widehat{\phi}_{\text {loc }}(\Omega)$ associée à la semi-norme $p_{i}$ et $B_{a^{\prime}}(0,2 \varepsilon)$ désigne la boule de rayon $2 \varepsilon$ de $\widehat{J \phi}(E)$ associée à la norme $\|\cdot\|_{\left\{p ! M_{a^{\prime} p}^{(\phi)}, 1, E\right\}}$. On choisit $i \in \mathbb{N}^{*}$ tel que $E \subset X_{i}$. 
Assertion. Soit $p \in \mathbb{N}$. Pour tout $\left(x^{\prime}, x^{\prime \prime}\right) \in \mathbb{R} \times \mathbb{R}^{n-1}$ on pose $f_{p}\left(\left(x^{\prime}, x^{\prime \prime}\right)\right)$ $=x^{\prime p}$ et $F_{p}=R_{E}\left(f_{p}\right)$. Alors $F_{p}$ est un jet appartenant $\grave{a} \widehat{J \phi}(E)$ et

$$
\forall a^{\prime}>0, \forall A>0, \quad\left\|F_{p}\right\|_{\left\{p ! M_{a^{\prime} p}^{(\phi)}, 1, E\right\}} \leq \frac{(2 A+\beta)^{p}}{h_{\left(M_{a^{\prime} k}^{(\phi)}\right)}(A)}
$$

en posant

$$
h_{\left(M_{a^{\prime} k}^{(\phi)}\right)}(A)=\inf _{k \geq 0} M_{a^{\prime} k}^{(\phi)} A^{k} .
$$

D'après $(28)$ et $(29)$, il existe une fonction $\theta_{p}$ appartenant à $\widehat{\phi}_{\text {loc }}(\Omega)$ telle que

$$
R_{E}\left(\theta_{p}\right)=F_{p}
$$

et

$$
\left\|\theta_{p}\right\|_{\left\{p ! M_{p / i}^{(\phi)}, 1, \bar{X}_{i}\right\}} \leq \frac{\left\|F_{p}\right\|_{\left\{p ! M_{a^{\prime} p}^{(\phi)}, 1, E\right\}}}{\varepsilon} \leq \frac{(2 A+\beta)^{p}}{\varepsilon h_{\left(M_{a^{\prime} k}^{(\phi)}\right)}(A)} .
$$

Supposons que $\phi$ ne soit pas non quasi-analytique. Alors, d'après le théorème de Denjoy-Carleman, il existe $r>0$ tel que la boule fermée $\bar{B}\left(y_{0}, r\right)$ soit contenue dans $X_{i}$ et, pour tout $x \in B(0, r)$, on ait $\theta_{p}(x)=x^{\prime p}$. D'après (31), pour tout entier $p$, on a

$$
(\beta+r)^{p} \leq \sup _{x \in \bar{X}_{i}}\left|\theta_{p}(x)\right| \leq\left\|\theta_{p}\right\|_{\left\{p ! M_{p / i}^{(\phi)}, 1, \bar{X}_{i}\right\}} \leq \frac{(2 A+\beta)^{p}}{\varepsilon h_{\left(M_{a^{\prime} k}^{(\phi)}\right)}(A)},
$$

ce qui est impossible si l'on impose $2 A<r$.

\section{Exemples}

6.1. Intersections à croissance modérée, intersection des classes de Gevrey. Dans tout ce paragraphe $\phi^{\prime}$ désigne la dérivée à droite de $\phi$.

6.1.1. Définition [3]. Soit $\left(M_{p}\right)_{p \geq 0}$ une suite de réels. On dit que la suite $\left(M_{p}\right)_{p \geq 0}$ est à croissance modérée lorsque

(i) $M_{0}=1$ et $\left(M_{p}\right)_{p \geq 0}$ est logarithmiquement convexe,

(ii) $\lim _{p \rightarrow+\infty} M_{p}^{1 / p}=+\infty$,

(iii) il existe $A>1$ tel que, pour tout entier $p$, on ait

$$
M_{p+1}^{p} \leq A^{p} M_{p}^{p+1} .
$$

On peut remarquer que, sous l'hypothèse (i), la condition (iii) est équivalente à la condition suivante :

(iv) il existe $A^{\prime}>1$ tel que, pour tout $(j, p) \in \mathbb{N}^{2}, 0 \leq j \leq p$, on ait

$$
M_{p} \leq A^{\prime p} M_{j} M_{p-j}
$$


Dans [3], les théorèmes d'extension ont été établis dans le cadre d'intersections de classes non quasi-analytiques à croissance modérée. L'intersection des classes de jets considérée est

$$
\widehat{J M}(E)=\bigcap_{a>0}\left\{p ! M_{p}^{a}, 1, E\right\}
$$

et l'intersection des classes de fonctions considérée est

$$
\widehat{M}=\bigcap_{a>0}\left\{p ! M_{p}^{a}, 1, \mathbb{R}^{n}\right\} .
$$

On peut démontrer aisément les deux lemmes suivants :

6.1.2. Lemme. Soit $\phi$ une fonction croissante sur $\mathbb{R}_{+}$, convexe et nulle en 0 . Les conditions suivantes sont équivalentes:

$$
\exists B_{1} \in \mathbb{R}_{+}, \forall x \in \mathbb{R}_{+}, \quad \phi(2 x) \leq B_{1} x+2 \phi(x)
$$

et

$$
\exists B_{2} \in \mathbb{R}_{+}, \forall x \in \mathbb{R}_{+}^{*}, \quad \phi^{\prime}(x) \leq \frac{\phi(x)}{x}+B_{2} .
$$

6.1.3. Lemme. Soit $\left(M_{p}\right)_{p \geq 0}$ une suite à croissance modérée. La fonction $\phi$, définie sur $\mathbb{R}_{+}$par $\phi(p)=\ln M_{p}$ et $\phi(p+(1-s))=s \phi(p)+$ $(1-s) \phi(p+1)$, pour tout entier $p$ et tout $s \in[0,1]$, est croissante, convexe, nulle en 0 et vérifie (32), (33) et $\lim _{t \rightarrow+\infty} f(t) / t=+\infty$.

6.1.4. Définition. Par analogie avec la définition précédente, on dit que $\phi$ est à croissance modérée lorsque $\phi$ est croissante sur $\mathbb{R}_{+}$, convexe, nulle en 0 et vérifie les conditions (32), (33) et $\lim _{t \rightarrow+\infty} f(t) / t=+\infty$.

6.1.5. PropriétÉ. Soit $\phi$ une fonction à croissance modérée. Alors, si $B_{2}$ désigne la constante donnée par (33), pour tout $a \geq 1$, on a

$$
\forall t \geq 0, \quad \phi(a t) \leq t B_{2} a \ln a+a \phi(t) .
$$

Démonstration. Soit $t \in \mathbb{R}_{+}^{*}$; notons $\phi_{t}(x)=\phi(t x)$. La relation (33) implique

$$
\forall x \in \mathbb{R}_{+}^{*}, \quad \phi_{t}^{\prime}(x) \leq \frac{\phi_{t}(x)}{x}+t B_{2},
$$

d'où

$$
\forall x \in \mathbb{R}_{+}^{*}, \quad \frac{x \phi_{t}^{\prime}(x)-\phi_{t}(x)}{x^{2}} \leq \frac{t B_{2}}{x} .
$$

Donc

$$
\forall a \geq 1, \quad \int_{1}^{a} \frac{x \phi_{t}^{\prime}(x)-\phi_{t}(x)}{x^{2}} d x \leq \int_{1}^{a} \frac{t B_{2}}{x} d x
$$

soit

$$
\forall a \geq 1, \quad \frac{\phi_{t}(a)}{a} \leq t B_{2} \ln a+\phi_{t}(1)
$$


d'où

$$
\forall a \geq 1, \quad \phi_{t}(a) \leq t B_{2} a \ln a+a \phi(t)
$$

Cette dernière inégalité est encore vraie pour $t=0$.

6.1.6. Corollaire. Soit $\left(M_{p}\right)_{p \geq 0}$ une suite à croissance modérée. Si $\phi$ est la fonction définie dans le lemme 6.1.3, alors $\widehat{J \phi}(E)=\widehat{J M}(E)$ et $\widehat{\phi}=\widehat{M}$.

6.2. Un exemple de suite à croissance non modérée. Soit $\phi$ une fonction à croissance modérée. En fixant $t=1$ dans (34), on a

$$
\forall a \geq 1, \quad \phi(a) \leq a \phi(1)+a B_{2} \ln a .
$$

Ceci démontre que $\widehat{J \phi}(E)$ est inclus dans l'intersection des classes de Gevrey. La fonction $\phi$ définie par $\phi(x)=x^{2}$ n'est pas à croissance modérée mais elle vérifie les hypothèses du théorème 4.1 . En ce sens, le théorème 4.1 généralise le théorème 8 de [3].

7. Remarques sur l'extension linéaire. Ici encore, $\phi$ désigne une fonction convexe et croissante sur $\mathbb{R}_{+}$vérifiant $\lim _{t \rightarrow+\infty} \phi(t) / t=+\infty$. Dans le théorème 4.1 , en général, l'extension de $\widehat{J \phi}(E)$ dans $\widehat{\phi}$ ne peut pas être réalisée à l'aide d'un opérateur linéaire.

7.1. Premier contre-exemple si $E=\{0\}$

Proposition. Il n'existe pas d'application linéaire continue $U: \widehat{J \phi}(\{0\})$ $\rightarrow \widehat{\phi}(\mathbb{R})$ telle que $R_{\{0\}} \circ U=\mathrm{id}_{\widehat{J \phi}}(\{0\})$.

Démonstration. On s'inspire des calculs de [10] et on suppose l'existence d'une telle application linéaire. Alors, il existe une suite $\left(\chi_{p}\right)_{p \geq 0}$ d'éléments de $\widehat{\phi}(\mathbb{R})$ telle que

$$
\forall(p, k) \in \mathbb{N}^{2}, \quad \chi_{p}^{(k)}(0)=\delta_{k, p}
$$

et

(36) $\forall a>0, \exists b>0, \exists C \geq 0, \forall p \in \mathbb{N}, \quad\left\|\chi_{p}\right\|_{\left\{p ! M_{a p}^{(\phi)}, 1, \mathbb{R}\right\}} \leq \frac{C}{p ! M_{b p}^{(\phi)}}$.

En particulier, avec $a=1$, il existe $b^{\prime}>0$ et $C^{\prime} \geq 0$ tels que

$$
\forall p \in \mathbb{N}, \quad \sup _{y \in \mathbb{R}_{+}}\left|\chi_{p}(y)\right| \leq \frac{C^{\prime}}{p ! M_{b^{\prime} p}^{(\phi)}} .
$$

D'après la formule de Taylor, pour tout $p \in \mathbb{N}$ et tout $x \in \mathbb{R}_{+}$, on a

$$
\left|\chi_{p}^{(p)}(x)-1\right| \leq \frac{x^{p}}{p !} \sup _{y \in \mathbb{R}_{+}}\left|\chi_{p}^{(2 p)}(y)\right|
$$


donc, d'après (36), avec $a=b^{\prime} / 2$, il existe $b>0$ et $C \geq 0$ tels que

$$
\forall p \in \mathbb{N}, \quad\left|\chi_{p}^{(p)}(x)-1\right| \leq \frac{x^{p}}{p !} \cdot \frac{C}{p ! M_{b p}^{(\phi)}}(2 p) ! M_{b^{\prime} p}^{(\phi)} .
$$

Soit $\tau_{p}$ tel que

$$
\tau_{p}^{p}=\frac{(p !)^{2} M_{b p}^{(\phi)}}{2 C(2 p) ! M_{b^{\prime} p}^{(\phi)}} .
$$

Pour tout $x \in\left[0, \tau_{p}\right]$, on a $\chi_{p}^{(p)}(x) \geq 1 / 2$. En intégrant $p$ fois, on obtient

$$
\frac{1}{2} \cdot \frac{x^{p}}{p !} \leq \chi_{p}(x)
$$

pour tout $x \in\left[0, \tau_{p}\right]$. Donc

$$
\frac{1}{2} \cdot \frac{\tau_{p}^{p}}{p !}=\frac{1}{4} \cdot \frac{(p !)^{2} M_{b p}^{(\phi)}}{p ! C(2 p) ! M_{b^{\prime} p}^{(\phi)}} \leq \chi_{p}\left(\tau_{p}\right) \leq \frac{C^{\prime}}{p ! M_{b^{\prime} p}^{(\phi)}},
$$

d'où

$$
M_{b^{\prime} p}^{(\phi)} M_{b p}^{(\phi)} \leq \frac{(2 p) !}{(p !)^{2}} 4 C C^{\prime} M_{b^{\prime} p}^{(\phi)}
$$

donc

$$
M_{b^{\prime} p}^{(\phi)} \frac{M_{b p}^{(\phi)}}{2^{2 p}} \leq 4 C C^{\prime} M_{b^{\prime} p}^{(\phi)} .
$$

Cette dernière inégalité est impossible puisque $M_{b p}^{(\phi)} / 4^{p}$ tend vers $+\infty$ lorsque $p$ tend vers $+\infty$.

7.2. Deuxième contre-exemple: les intersections de classes à croissance modérée. Dans ce paragraphe, $\widehat{\phi}([0,1+\varepsilon])$ désigne l'ensemble des fonctions de classe $C^{\infty}$ sur $[0,1+\varepsilon]$ dont le jet appartient à $\widehat{J \phi}([0,1+\varepsilon])$.

Proposition. Soit $\phi$ une fonction à croissance modérée et soit $\varepsilon>0$. Il n'existe pas d'application linéaire continue $U: \widehat{J \phi}([0,1]) \rightarrow \widehat{\phi}([0,1+\varepsilon])$ telle que $R_{[0,1]} \circ U=\mathrm{id}_{\widehat{J \phi}}([0,1])$.

Démonstration. On rappelle que $\phi^{\prime}$ désigne la dérivée à droite de $\phi$. Soit $b>0$; la fonction $h_{\left(M_{b k}\right)}^{(\phi)}$ est définie comme dans (30) par

$$
\forall r>0, \quad h_{\left(M_{b k}^{(\phi)}\right)}(r)=\inf _{k \in \mathbb{N}} M_{b k}^{(\phi)} r^{k} .
$$

On définit également la fonction $h_{\phi, b}$ par

$$
\begin{aligned}
\forall r>0, \quad h_{\phi, b}(r) & =\inf _{t \geq 0} M_{b t}^{(\phi)} r^{t}=\inf _{t \geq 0}\left\{\exp (\phi(b t)) r^{t}\right\} \\
& =\exp \left(\inf _{t \geq 0}\{\phi b(t)+t \ln r\}\right) .
\end{aligned}
$$


Pour tout $r>0$, on a

$$
h_{\left(M_{b k}^{(\phi)}\right)}(r) \geq h_{\phi, b}(r) .
$$

Supposons qu'il existe une application linéaire continue $U: \widehat{J \phi}([0,1]) \rightarrow$ $\widehat{\phi}([0,1+\varepsilon])$ telle que $R_{[0,1]} \circ U=\operatorname{id}_{\widehat{J \phi}}([0,1])$. Alors pour tout $a>0$, on a

$$
\begin{aligned}
\exists b>0, \exists C \geq 0, \forall F \in \widehat{J \phi}([0,1]), \\
\|U(F)\|_{\left\{p ! M_{a p}^{(\phi)}, 1,[0,1+\varepsilon]\right\}} \leq C\|F\|_{\left\{p ! M_{b p}^{(\phi)}, 1,[0,1]\right\}}
\end{aligned}
$$

Dans ce qui suit $a$ est un réel strictement positif; on note $b$ et $C$ les deux réels associés à $a$ par (37). Si $F_{j}$ est le jet sur $[0,1]$ de la fonction $x \mapsto x^{j}$, en notant $\left\|F_{j}\right\|_{b}=\left\|F_{j}\right\|_{\left\{p ! M_{b p}^{(\phi)}, 1,[0,1]\right\}}$, pour tout $A>0$, on a

$$
\left\|F_{j}\right\|_{b} \leq \sup _{0 \leq k \leq j} \frac{2^{k} j ! A^{k}}{(j-k) ! k ! A^{k} M_{b k}^{(\phi)}} \leq \frac{(1+2 A)^{j}}{h_{\left(M_{b k}^{(\phi)}\right)}(A)} \leq \frac{(1+2 A)^{j}}{h_{\phi, b}(A)} .
$$

Ainsi, pour tout entier $j$, on a

$$
\begin{aligned}
\left\|F_{j}\right\|_{b} & \leq \exp \left(j \ln (1+2 A)-\inf _{t \geq 0}\{\phi(b t)+t \ln A\}\right) \\
& \leq \exp \left(2 j A-\inf _{t \geq 0}\{\phi(b t)+t \ln A\}\right)
\end{aligned}
$$

Si $t_{0}$ et $A$ vérifient $A=\exp \left(-b \phi^{\prime}\left(b t_{0}\right)\right)$, on a

$$
\left\|F_{j}\right\|_{b} \leq \exp \left[2 j \exp \left(-b \phi^{\prime}\left(b t_{0}\right)\right)-\left(\phi\left(b t_{0}\right)-t_{0} b \phi^{\prime}\left(b t_{0}\right)\right)\right] .
$$

Soit $\alpha>0$. D'après (37), en posant

on a

$$
f_{j}=U\left(F_{j}\right) \quad \text { et } \quad\left\|f_{j}\right\|_{\alpha}=\left\|f_{j}\right\|_{\left\{p ! M_{\alpha p}^{(\phi)}, 1,[0,1+\varepsilon]\right\}},
$$

$$
\exists \beta>0, \exists C(\alpha) \geq 0, \forall j \in \mathbb{N}, \quad\left\|f_{j}\right\|_{\alpha} \leq C(\alpha)\left\|F_{j}\right\|_{\beta} .
$$

On effectue un développement de Taylor de la fonction $f_{j}^{(j)}$, à l'ordre $j$, entre 1 et $x \in[1,1+\varepsilon]$. En utilisant $(38)$ et en posant $\widetilde{C}(\alpha)=C(\alpha) / h_{\phi, \beta}(1 / 2)$, on obtient

$$
\left|f_{j}^{(j)}(x)-j !\right| \leq \frac{(x-1)^{j}}{j !}(2 j) ! M_{2 \alpha j}^{(\phi)} C(\alpha)\left\|F_{j}\right\|_{\beta} \leq(x-1)^{j} \widetilde{C}(\alpha) j ! M_{2 \alpha j}^{(\phi)} 2^{3 j} .
$$

Soit $\tau_{j}=1 /\left(8\left(2 \widetilde{C}(\alpha) M_{2 \alpha j}^{(\phi)}\right)^{1 / j}\right)$. De $\lim _{j \rightarrow+\infty} \tau_{j}=0$, on déduit que $1+\tau_{j} \leq$ $1+\varepsilon$ pour $j$ assez grand, et donc $f_{j}^{(j)}(x) \geq j ! / 2$ pour tout $x \in\left[1,1+\tau_{j}\right]$. Comme cette inégalité est encore vraie sur $[0,1]$, en intégrant $j$ fois, si $j$ est assez grand, on obtient

$$
\begin{aligned}
f_{j}\left(1+\tau_{j}\right) & \geq \frac{1}{2} \exp \left[j \ln \left(1+\tau_{j}\right)\right] \\
& \geq \frac{1}{2} \exp \left[\frac{j \tau_{j}}{2}\right]=\frac{1}{2} \exp \left[j \frac{1}{16\left(2 \widetilde{C}(\alpha) M_{2 \alpha j}^{(\phi)}\right)^{1 / j}}\right] .
\end{aligned}
$$


Par ailleurs, $f_{j}\left(1+\tau_{j}\right) \leq\left\|f_{j}\right\|_{a} \leq C\left\|F_{j}\right\|_{b}$ et, si $j$ est assez grand,

$$
\frac{1}{16(2 \widetilde{C}(\alpha))^{1 / j}\left(M_{2 \alpha j}^{(\phi)}\right)^{1 / j}} \geq \frac{1}{20\left(M_{2 \alpha j}^{(\phi)}\right)^{1 / j}}
$$

$\left(\operatorname{car} \lim _{j \rightarrow+\infty}(2 \widetilde{C}(\alpha))^{1 / j}=1\right)$. Donc, en utilisant (39) et (40), pour $j$ assez grand, on a

$$
\begin{aligned}
& \ln \frac{1}{2}+\frac{j}{20 \exp (\phi(2 \alpha j) / j)} \\
& \quad \leq 2 j \exp \left(-b \phi^{\prime}\left(b t_{0}\right)\right)-\left(\phi\left(b t_{0}\right)-t_{0} b \phi^{\prime}\left(b t_{0}\right)\right)+\ln C .
\end{aligned}
$$

D'autre part, en utilisant la condition de croissance modérée (33) et l'inégalité $\phi^{\prime}\left(b t_{0}\right) \geq \phi\left(b t_{0}\right) /\left(b t_{0}\right)$, on a

$$
2 j \exp \left(-b \phi^{\prime}\left(b t_{0}\right)\right)-\left(\phi\left(b t_{0}\right)-t_{0} b \phi^{\prime}\left(b t_{0}\right)\right) \leq 2 j \exp \left(-\frac{\phi\left(b t_{0}\right)}{t_{0}}\right)+B_{2} b t_{0} .
$$

Donc, avec $2 j=\exp \left(\phi\left(b t_{0}\right) / t_{0}\right) B_{2} b t_{0}$, l'inégalité (41) implique

$$
\frac{j}{20 \exp (\phi(2 \alpha j) / j)} \leq \ln 2+2 B_{2} b t_{0}+\ln C \text {. }
$$

Si $2 B_{2} b t_{0}$ est assez grand, ce qui équivaut à $j$ assez grand, on a

$$
2 B_{2} b t_{0}+\ln 2+\ln C \leq 4 B_{2} b t_{0} .
$$

Donc l'inégalité précédente implique

$$
\frac{\phi\left(b t_{0}\right)}{t_{0}} \leq \frac{\phi\left(\alpha B_{2} b t_{0} \exp \left(\phi\left(b t_{0}\right) / t_{0}\right)\right)}{\left[\exp \left(\phi\left(b t_{0}\right) / t_{0}\right) B_{2} b t_{0} / 2\right]}+\ln 160 .
$$

Or, d'après (34) avec $a=\exp \left(\phi\left(b t_{0}\right) / t_{0}\right)$ et $t=\alpha B_{2} b t_{0}$, on a

$$
\frac{\phi\left(\alpha B_{2} b t_{0} \exp \left(\phi\left(b t_{0}\right) / t_{0}\right)\right)}{\exp \left(\phi\left(b t_{0}\right) / t_{0}\right) B_{2} b t_{0}} \leq \frac{\phi\left(\alpha B_{2} b t_{0}\right)}{B_{2} b t_{0}}+B_{3} \alpha \frac{\phi\left(b t_{0}\right)}{t_{0}} .
$$

Donc

$$
\frac{\phi\left(b t_{0}\right)}{t_{0}} \leq \ln 160+2 \frac{\phi\left(\alpha B_{2} b t_{0}\right)}{B_{2} b t_{0}}+2 B_{3} \alpha \frac{\phi\left(b t_{0}\right)}{t_{0}} .
$$

Si l'on choisit $\alpha$ tel que $2 B_{3} \alpha<1 / 4$ et $\alpha B_{2}<1 / 4$, cette inégalité est impossible lorsque $t_{0}$ tend vers $+\infty$, c'est-à-dire lorsque $j$ tend vers $+\infty$.

REMARQUE. Si $\phi$ est une fonction à croissance modérée, alors le quotient $\phi(u) /(u \ln u)$ est borné au voisinage de $+\infty$. Dans le paragraphe suivant, on démontre que, pour de "bons compacts", on obtient un résultat d'extension linéaire lorsque le quotient $\phi(u) /(u \ln u)$ a pour limite $+\infty$ lorsque $u$ tend vers $+\infty$.

8. Extension linéaire. On utilise ici, dans le cadre des intersections, la méthode développée par W. Pleśniak dans [11]. 
8.1. Notations. Pour tout entier $k, P_{k}$ désigne l'ensemble des polynômes de degré inférieur ou égal à $k$ et $P_{-1}=\{0\}$. Si $E$ est un compact de $\mathbb{R}^{n}$ et si $f \in C(E)$ on pose $\|f\|_{E}=\sup _{x \in E}|f(x)|$ et $\operatorname{dist}_{E}\left(f, P_{k}\right)=$ $\inf \left\{\|f-P\|_{E}: P \in P_{k}\right\}$.

8.2. Proposition [11]. Soit $\mathbf{P}$ un cube compact de $\mathbb{R}^{n}$. Il existe une constante $A$ ne dépendant que de $\mathbf{P}$ et de $n$ telle que, pour tout $t \in] 0,1[$ et pour toute fonction $f \in C^{\infty}\left(\mathbb{R}^{n}\right)$, on ait

$$
\forall p \in \mathbb{N}, \forall k \geq \frac{n p}{t}, \quad(k+2)^{p} \operatorname{dist}_{\mathbf{P}}\left(f, P_{k}\right) \leq\left(\frac{A}{1-t}\right)^{p}(k+1)^{n} \max _{1 \leq j \leq n}\left\|\frac{\partial^{p} f}{\partial x_{j}^{p}}\right\|_{\mathbf{P}} .
$$

8.3. Définitions et propriétés. (i) Soient $\left(M_{p}\right)$ une suite non analytique, $E$ un compact de $\mathbb{R}^{n}$ et $C_{2}>0$. On pose

$$
\mathbf{A}\left\{p ! M_{p}, C_{2}, E\right\}=\left\{f \in C(E): \sup _{p \geq 0} \sup _{k \geq-1} \frac{(k+2)^{p} \operatorname{dist}_{E}\left(f, P_{k}\right)}{p ! M_{p} C_{2}^{p}}<+\infty\right\} ;
$$

muni de la norme $\|\cdot\|_{\mathbf{A}\left\{p ! M_{p}, C_{2}, E\right\}}$ définie par

$$
\|f\|_{\mathbf{A}\left\{p ! M_{p}, C_{2}, E\right\}}=\sup _{p \geq 0} \sup _{k \geq-1} \frac{(k+2)^{p} \operatorname{dist}_{E}\left(f, P_{k}\right)}{p ! M_{p} C_{2}^{p}},
$$

l'espace $\mathbf{A}\left\{p ! M_{p}, C_{2}, E\right\}$ est un espace de Banach.

(ii) On pose

$$
\mathbf{A}\{\phi, E\}=\bigcap_{a>0} \mathbf{A}\left\{p ! M_{a p}^{(\phi)}, 1, E\right\}
$$

muni de la famille de normes $\left(\|\cdot\|_{\mathbf{A}\left\{p ! M_{a p}^{(\phi)}, 1, E\right\}}\right)_{a>0}$, l'espace $\mathbf{A}\{\phi, E\}$ est un espace de Fréchet.

8.4. Proposition. Il existe une constante $\delta>0$ ne dépendant que de $n$ et de $E$ telle que, pour tout $\gamma_{1} \geq 1$, l'application

$$
\Gamma_{E}:\left\{p ! M_{a p}^{(\phi)}, \gamma_{1}, \mathbb{R}^{n}\right\} \rightarrow \mathbf{A}\left\{p ! M_{a(p+n)}^{(\phi)}, \delta \gamma_{1}, E\right\}, \quad f \mapsto f_{\mid E},
$$

soit bien définie et continue.

Démonstration. On considère un cube compact $\mathbf{P}$ contenant $E$. Soit $p \in \mathbb{N}$.

(i) En appliquant la proposition 8.2, avec $t=1 / 2$ et $p+n$ à la place de $p$, pour tout $k \geq 2 n(p+n)$, on a

$$
(k+2)^{p} \operatorname{dist}_{\mathbf{P}}\left(f, P_{k}\right) \leq\left(4^{n} n ! A^{n} \gamma_{1}^{n}\right)\left(4 A \gamma_{1}\right)^{p} p ! M_{a(p+n)}^{(\phi)}\|f\|_{\left\{p ! M_{a p}^{(\phi)}, \gamma_{1}, \mathbb{R}^{n}\right\}} .
$$

(ii) $\mathrm{Si}-1 \leq k<2 n(p+n)$ on a

$$
(k+2)^{p} \operatorname{dist}_{\mathbf{P}}\left(f, P_{k}\right) \leq(k+2)^{p}\|f\|_{E} \leq\left(2 n p+2 n^{2}+1\right)^{p}\|f\|_{E} .
$$


Or

$$
\left(2 n p+2 n^{2}+1\right)^{p}=\left(2 n^{2}\right)^{p}\left(\frac{p}{n}+1+\frac{1}{2 n^{2}}\right)^{p} \leq\left(2 n^{2}\right)^{p}(p+2)^{p} \leq e\left(4 n^{2} e\right)^{p} p !
$$

et donc

$$
(k+2)^{p} \operatorname{dist}_{\mathbf{P}}\left(f, P_{k}\right) \leq e\left(4 n^{2} e\right)^{p}\|f\|_{\left\{p ! M_{a p}^{(\phi)}, \gamma_{1}, \mathbb{R}^{n}\right\}} p ! M_{a(p+n)}^{(\phi)} .
$$

Posons $\delta=\max \left\{4 n^{2} e, 4 A\right\}$. Alors

$$
f \in \mathbf{A}\left\{p ! M_{a(p+n)}^{(\phi)}, \delta \gamma_{1}, E\right\}
$$

et

$$
\|f\|_{\mathbf{A}\left\{p ! M_{a(p+n)}^{(\phi)}, \delta \gamma_{1}, E\right\}} \leq \max \left\{4^{n} n ! A^{n} \gamma_{1}^{n}, e\right\}\|f\|_{\left\{p ! M_{a p}^{(\phi)}, \gamma_{1}, \mathbb{R}^{n}\right\}} .
$$

Par un argument similaire à celui terminant la démonstration du théorème 4.1 on établit :

8.5. Corollaire. Notons encore $\Gamma_{E}$ l'application de restriction

$$
\widehat{\phi} \rightarrow \mathbf{A}\{\phi, E\}, \quad f \mapsto f_{\mid E} .
$$

Cette application est bien définie et continue.

8.6. Propriété de Markov et polynômes d'interpolation de Lagrange ([9], [11], [12])

8.6.1. Propriété de Markov pour un compact. Soient $E$ un compact de $\mathbb{R}^{n}$ et $r$ un réel supérieur ou égal à 2. On dit que $E$ a la propriété de Markov $\mathbf{M}(r)$ lorsqu'il existe une constante $\mathcal{M}>0$ telle que, pour tout polynôme $Q$ et pour tout multi-indice $J$ de $\mathbb{N}^{n}$, on ait $\left\|D^{J} Q\right\|_{E} \leq \mathcal{M}(\operatorname{deg} Q)^{r|J|}\|Q\|_{E}$.

Cette propriété implique la propriété suivante :

Il existe une constante $\widetilde{\mathcal{M}}>0$ telle que, pour tout polynôme $Q$ et pour tout $x \in \mathbb{R}^{n}$ tel que $d(x, E) \leq 1 /(\operatorname{deg} Q)^{r}$, on ait $|Q(x)| \leq \widetilde{\mathcal{M}}\|Q\|_{E}$.

Le lemme suivant est une variante du lemme 3.1.1.

8.6.2. Lemme. Soient $E$ un compact de $\mathbb{R}^{n},\left(M_{p}^{\prime}\right)_{p \geq 0}$ une suite non quasi-analytique et $\left(e_{k}\right)_{k \geq 0}$ une suite de réels strictement positifs. Il existe une suite $\left(u_{k}\right)_{k \geq 0}$ de fonctions $C^{\infty}$ sur $\mathbb{R}^{n}$ telle que

- $\forall k \in \mathbb{N}, 0 \leq u_{k} \leq 1$,

- $\forall k \in \mathbb{N}, u_{k}=1$ au voisinage de $E$,

- $\forall k \in \mathbb{N}, \operatorname{supp}\left(u_{k}\right) \subset\left\{x \in \mathbb{R}^{n}: d(x, E) \leq e_{k}\right\}$,

- $\forall J \in \mathbb{N}^{n}, \forall x \in \mathbb{R}^{n}$,

$$
\left|D^{J} u_{k}(x)\right| \leq\left(C d^{\prime} / e_{k}\right)^{|J|}|J| ! M_{|J|}^{\prime}
$$

où $d^{\prime}=\sum_{p \geq 1} M_{p-1}^{\prime} /\left(p M_{p}^{\prime}\right)$ et $C$ est une constante qui ne dépend que de la dimension $n$. 
8.6.3. Polynômes d'interpolation de Lagrange ([9], [14]). Soit $\varkappa: \mathbb{N}^{*} \rightarrow$ $\mathbb{N}^{n}$ une bijection telle que pour tout $j \in \mathbb{N}^{*}$ on ait $|\varkappa(j)| \leq|\varkappa(j+1)|$. Pour tout entier $j \in \mathbb{N}^{*}$, on pose $s_{j}=x^{\varkappa(j)}$. On peut vérifier que $\left(s_{j}\right)_{1 \leq i \leq\left(\begin{array}{c}n+k \\ k\end{array}\right)}$ est une base de $P_{k}$ pour tout entier $k$. Si $t^{(k)}=\left\{t_{1}, \ldots, t_{k}\right\}$ est un ensemble de $k$ points de $\mathbb{R}^{n}$, on note

$$
V\left(t^{(k)}\right)=V\left(t_{1}, \ldots, t_{k}\right)=\operatorname{det}\left(s_{j}\left(t_{l}\right)\right)_{1 \leq j, l \leq k}
$$

le déterminant de Vandermonde associé à $t^{(k)}$. Si $V\left(t^{(k)}\right) \neq 0$ on pose

$$
L^{j}\left(x, t^{(k)}\right)=\frac{V\left(t_{1}, \ldots, t_{j-1}, x, t_{j+1}, \ldots, t_{k}\right)}{V\left(t^{(k)}\right)} .
$$

Un ensemble $t^{(k)}=\left\{t_{1}, \ldots, t_{k}\right\}$ de $k$ points du compact $E$ est appelé un système de points extrémaux de Fekete sur $E$ d'ordre $k$ lorsque $V\left(t^{(k)}\right) \geq$ $V\left(r^{(k)}\right)$ pour tout $r^{(k)}=\left\{r_{1}, \ldots, r_{k}\right\} \subset E$.

8.6.4. DÉfinition. Soit $E$ un compact de $\mathbb{R}^{n}$. On dit que $E$ est unisolvant si, pour tout polynôme $p, p_{\mid E}=0$ implique $p=0$. On dit que $E$ est $C^{\infty}$ déterminant si, pour toute fonction $f \in C^{\infty}\left(\mathbb{R}^{n}\right), f_{\mid E}=0$ implique $f_{\mid E}^{J}=0$ pour tout multi-indice $J$.

8.6.5. PropriÉté [12]. Si E a la propriété de Markov, alors E est $C^{\infty}$ déterminant et donc unisolvant.

8.6.6. Propriété [14]. Si E est unisolvant alors, pour tout entier $l>0$, il existe $\left\{x_{1}, \ldots, x_{l}\right\} \subset E$ tel que $V\left(x_{1}, \ldots, x_{l}\right)>0$.

8.6.7. Définition ([9], [14]). Soit $t^{(k)}=\left\{t_{1}, \ldots, t_{k}\right\}$ un système de points extrémaux de Fekete sur $E$ d'ordre $k$ et soit $f \in C(E)$. Le polynôme

$$
L_{t^{(k)}} f(x)=\sum_{j=1}^{\left(\begin{array}{c}
n+k \\
k
\end{array}\right)} f\left(t_{j}\right) L^{j}\left(x, t^{(k)}\right)
$$

est appelé le polynôme d'interpolation de $f$ associé à $t^{(k)}$.

Si $E$ a la propriété de Markov, à tout entier $k>0$ on associe un système $t^{(k)}$ de points extrémaux de Fekete sur $E$ d'ordre $k$ et on note $L_{k} f(x)$ le polynôme $L_{t^{(k)}} f(x)$.

8.6.8. Propriété [9]. Soit $E$ un compact de $\mathbb{R}^{n}$ unisolvant. Avec les notations précédentes, si $f \in C(E)$ on a

$$
\forall k \in \mathbb{N}^{*}, \quad\left\|f-L_{k} f\right\|_{E} \leq 2(k+1)^{n} \operatorname{dist}_{E}\left(f, P_{k}\right)
$$

et donc

$$
\forall k \in \mathbb{N}^{*}, \quad\left\|L_{k+1} f-L_{k} f\right\|_{E} \leq 4(k+2)^{n} \operatorname{dist}_{E}\left(f, P_{k}\right) .
$$


8.7. ThÉORÈme. Soit $E$ un compact de $\mathbb{R}^{n}$ vérifiant la propriété de Markov $\mathbf{M}(r)$. On suppose que $\phi$ vérifie la condition suivante:

$$
\lim _{u \rightarrow+\infty} \frac{\phi(u)}{u \ln u}=+\infty \text {. }
$$

Alors il existe une application linéaire continue $L: \mathbf{A}\{\phi, E\} \rightarrow \widehat{\phi}$ telle que $\Gamma_{E} \circ L=\operatorname{id}_{\mathbf{A}\{\phi, E\}}$.

Remarques. (i) La condition (42) est équivalente à la condition

$$
\forall a>0, \quad \lim _{t \rightarrow+\infty} \frac{\exp \phi(a t)}{t^{t}}=+\infty .
$$

(ii) La condition (43) implique clairement

$$
\forall a>0, \quad \lim _{p \rightarrow+\infty} \frac{M_{a p}^{(\phi)}}{p !}=+\infty
$$

et la condition de non quasi-analyticité (15).

Démonstration du théorème 8.7. On applique la proposition 4.2 avec la suite $\left(u_{p}\right)_{p \geq 0}$ définie par $u_{p}=1$ pour tout $p \geq 0$ et on note $\left(M_{p}^{\prime}\right)_{p \geq 0}$ la suite $\left(M_{p}^{(\psi)}\right)_{p \geq 0}$ obtenue. On applique le lemme précédent avec $e_{k}=1 /(k+1)^{r}$. Comme précédemment, à tout entier $k>0$ on associe un système $t^{(k)}$ de points extrémaux de Fekete sur $E$ d'ordre $k$. Soit $f \in \mathbf{A}\{\phi, E\}$; on note $L_{k} f=L_{t^{(k)}} f(x)$. On pose enfin

$$
\forall x \in \mathbb{R}^{n}, \quad L(f)(x)=u_{1}(x) L_{1} f(x)+\sum_{k \geq 1} u_{k}(x)\left(L_{k+1} f(x)-L_{k} f(x)\right) .
$$

Soit $P$ un multi-indice de longueur $p$. Pour tout $x \in \mathbb{R}^{n}$ posons

$$
F_{k}^{P}(x)=D^{P}\left[u_{k}(x)\left(L_{k+1} f(x)-L_{k} f(x)\right)\right] .
$$

Alors, en utilisant les notations du lemme 8.6.2, comme dans [11], proposition 3.3 , on a

$$
\begin{aligned}
\sup _{x \in \mathbb{R}^{n}}\left|F_{k}^{P}(x)\right| \leq & 4 \mathcal{M} \widetilde{\mathcal{M}} p ! M_{p}^{\prime} \\
& \times \sum_{L ; L \leq P}\left(\begin{array}{l}
P \\
L
\end{array}\right)\left(C d^{\prime}\right)^{l}(k+2)^{r p+n+2} \frac{1}{(k+2)^{2}} \operatorname{dist}_{E}\left(f, P_{k}\right) .
\end{aligned}
$$

D'après 8.3 , pour tout $a>0$, on a

$$
\operatorname{dist}_{E}\left(f, P_{k}\right) \leq(k+2)^{-(r p+n+2)}\|f\|_{\mathbf{A}\left\{p ! M_{a p}^{(\phi)}, 1, E\right\}}(r p+n+2) ! M_{a(r p+n+2)}^{(\phi)} .
$$

De plus, avec les notations de la proposition 4.2 on a

$$
\forall l \in \mathbb{N}, \quad M_{l}^{\prime} \leq C_{2}(a) M_{a l}^{(\phi)},
$$

donc, en supposant $C d^{\prime} \geq 1$, on a 


$$
\begin{aligned}
\sup _{x \in \mathbb{R}^{n}}\left|F_{k}^{P}(x)\right| \leq & 4 \mathcal{M} \widetilde{\mathcal{M}}\left(2 C d^{\prime}\right)^{p} \frac{1}{(k+2)^{2}} C_{2}(a) M_{0}^{(\phi)} \\
& \times\|f\|_{\mathbf{A}\left\{p ! M_{a p}^{(\phi)}, 1, E\right\}}((r+1) p+n+2) ! M_{a((r+1) p+n+2)}^{(\phi)} .
\end{aligned}
$$

Donc $\sum_{k \geq 0} F_{k}^{P}$ converge uniformément sur $\mathbb{R}^{n}$. De plus, pour tout $a>0$, on a

$$
\begin{aligned}
\sup _{x \in \mathbb{R}^{n}}\left|\sum_{k \geq 1} F_{k}^{P}(x)\right| \leq & \tau_{1}(a)\left(2 C d^{\prime}\right)^{p} \\
& \times\|f\|_{\mathbf{A}\left\{p ! M_{a p}^{(\phi)}, 1, E\right\}}((r+1) p+n+2) ! M_{a((r+1) p+n+2)}^{(\phi)}
\end{aligned}
$$

en posant

$$
\tau_{1}(a)=4 \mathcal{M} \widetilde{\mathcal{M}} C_{2}(a) M_{0}^{(\phi)} \sum_{k \geq 1} \frac{1}{(k+2)^{2}} .
$$

Pour tout entier $p$, on a

$$
\left(M_{2 a(r+1) p}^{(\phi)}\right)^{2} \leq M_{0}^{(\phi)} M_{4 a(r+1) p}^{(\phi)}
$$

Pour tout entier $p \geq(n+2) /(r+1)$, on a

$$
M_{a((r+1) p+n+2)}^{(\phi)} \leq M_{2 a(r+1) p}^{(\phi)} \leq M_{0}^{(\phi)} \frac{1}{M_{2 a(r+1) p}^{(\phi)}} M_{4 a(r+1) p}^{(\phi)} .
$$

De (44), on déduit qu'il existe $p_{0}(a) \in \mathbb{N}$ tel que

$$
\forall p \geq p_{0}(a), \quad \frac{\left(2 C d^{\prime}\right)^{p}((r+1) p+n+2) !}{\left(M_{a(r+1) p}^{(\phi)}\right)^{2}} M_{0}^{(\phi)} \leq 1 .
$$

Ainsi, pour tout $p \geq \max \left\{p_{0}(a),(n+2) /(r+1)\right\}$, on a

$$
\left(2 C d^{\prime}\right)^{p}((r+1) p+n+2) ! M_{a((r+1) p+n+2)}^{(\phi)} \leq M_{0}^{(\phi)} M_{4 a(r+1) p}^{(\phi)} .
$$

Il en résulte que

$$
L(f) \in\left\{p ! M_{4 a(r+1) p}^{(\phi)}, 1, \mathbb{R}^{n}\right\}
$$

De plus, si

$$
\stackrel{\Gamma(a)}{=} \max _{p ; p<\max \left\{p_{0}(a),(n+2) /(r+1)\right\}}\left\{\frac{\left(2 C d^{\prime}\right)^{p}((r+1) p+n+2) ! M_{a((r+1) p+n+2)}^{(\phi)}}{p ! M_{4 a(r+1) p}^{(\phi)}}\right\},
$$

alors

$$
\|L(f)\|_{\left\{p ! M_{4 a(r+1) p}^{(\phi)}, 1, \mathbb{R}^{n}\right\}} \leq \max \left\{\Gamma(a), M_{0}^{(\phi)}\right\} \tau_{1}(a)\|f\|_{\mathbf{A}\left\{p ! M_{a p}^{(\phi)}, 1, E\right\}},
$$

ceci pour tout réel $a>0$, ce qui démontre le théorème.

Remarque. L'application $\Gamma_{E}: \widehat{\phi} \rightarrow \mathbf{A}\{\phi, E\}$ est surjective. 
8.8. ThÉORÈme. Soit $E$ un compact de $\mathbb{R}^{n}$ vérifiant la propriété de Markov $\mathbf{M}(r)$. On suppose que $\phi$ vérifie la condition (42). Alors il existe une application linéaire continue $U: \widehat{J \phi}(E) \rightarrow \widehat{\phi}$ telle que $R_{E} \circ U=\mathrm{id}_{\widehat{J \phi}}(E)$. De plus les espaces $\widehat{J \phi}(E)$ et $\mathbf{A}\{\phi, E\}$ sont isomorphes.

Démonstration. Soit $F \in \widehat{J \phi}(E)$. D'après le théorème 4.1, il existe une fonction $f \in \widehat{\phi}$ telle que $F=R_{E}(f)$. On a alors $\Gamma_{E}(f)=F^{0}$. Donc l'application

$$
\Lambda: \widehat{J \phi}(E) \rightarrow \mathbf{A}\{\phi, E\}, \quad\left(F^{L}\right)_{L \in \mathbb{N}^{n}} \mapsto F^{0},
$$

est bien définie. On a donc le diagramme suivant :

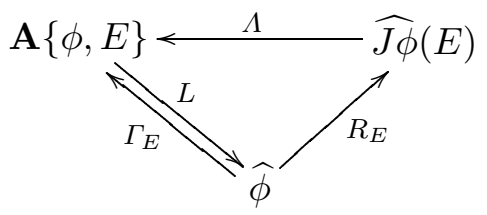

$\Gamma_{E}=\Lambda \circ R_{E}$ est surjective, donc $\Lambda$ est surjective. D'après la propriété 8.6.5, $E$ est $C^{\infty}$ déterminant. Il en résulte que $\Lambda$ est injective. On note $F_{\widehat{\phi}}(E)=$ $\left\{f \in \widehat{\phi}:\left(f_{\mid E}^{J}\right)_{J \in \mathbb{N}^{n}}=0\right\}$. Alors le théorème 4.1 implique $\widehat{J \phi}(E)=\widehat{\phi} / F_{\widehat{\phi}}(E)$. Ainsi $\Lambda$ est l'application quotient de $\Gamma_{E}$ :

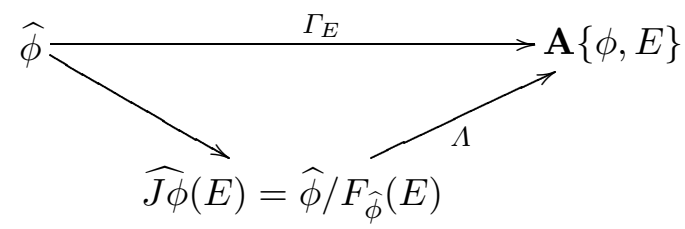

$\Gamma_{E}$ est continue et, puisque $E$ est $C^{\infty}$ déterminant, on a

$$
\operatorname{ker}\left(\Gamma_{E}\right)=\left\{f \in \widehat{\phi}: f_{\mid E}=0\right\}=F_{\widehat{\phi}}(E) .
$$

Donc $\Lambda$ est continue et, d'après le théorème de l'application ouverte, $\Lambda$ est une application bicontinue.

Remarque. Le théorème précédent généralise un théorème de U. Franken ([6], théorème 1). Dans [6], les hypothèses impliquent que, pour un $\alpha>1$, le quotient $\phi(u) / u^{\alpha}$ tend vers $+\infty$ lorsque $u$ tend vers $+\infty$. C'est une condition plus restrictive que la condition (42). Ici, contrairement à [6], la démonstration donne une "construction explicite" de l'application linéaire d'extension.

9. Extension analytique. Dans tout ce paragraphe $\phi$ désigne une fonction non quasi-analytique telle que $\phi(0)=0$. Ensuite $\Omega$ désigne un ouvert de $\mathbb{R}^{n}$ de frontière $\partial \Omega$ et $E$ un compact de $\mathbb{R}^{n}$. Si $\Omega=\mathbb{R}^{n}$ on pose $\Omega^{*}=\mathbb{C}^{n}$, sinon on pose $\Omega^{*}=\left\{u+i v: u \in \Omega, v \in \mathbb{R}^{n},|v|<d(u, \partial \Omega)\right\}$. Pour étendre 
les jets appartenant à $\widehat{J \phi}(E)$ en des fonctions réelles analytiques sur $\mathbb{R}^{n} \backslash E$, on reprend la méthode développée par M. Valdivia dans [17]. On obtient aisément le théorème suivant et ses deux corollaires.

9.1. ThÉORÈME. Il existe un opérateur linéaire continu $T: \widehat{\phi}(\Omega) \rightarrow$ $\widehat{\phi}(\Omega)$ vérifiant les conditions suivantes:

(i) Pour toute fonction $f \in \widehat{\phi}(\Omega)$, la fonction $T(f)$ s'étend en une fonction holomorphe sur $\Omega^{*}$.

(ii) Pour tous $\varepsilon>0, s \in \mathbb{N}^{*}$ et $f \in \widehat{\phi}(\Omega)$, il existe un compact $H$ de $\Omega$ tel que $\left|D^{J} T(f)(x)-D^{J} f(x)\right| \leq \varepsilon$ pour tout $x \in \Omega \backslash H$ et tout multi-indice $J$ de longueur $j \leq s$.

9.2. Corollaire. Soit $E$ un ensemble compact non vide de $\mathbb{R}^{n}$. Notons $R_{E}$ l'opérateur de restriction défini au 2.3.5. S'il existe une application linéaire continue $U: \widehat{J \phi}(E) \rightarrow \widehat{\phi}\left(\mathbb{R}^{n}\right)$ telle que $R_{E} \circ U=\operatorname{id}_{\widehat{J \phi}}(E)$, alors il existe une application linéaire continue $U_{1}: \widehat{J \phi}(E) \rightarrow \widehat{\phi}\left(\mathbb{R}^{n}\right)$ telle que $U_{1}(F)$ soit réelle analytique sur $\mathbb{R}^{n} \backslash E$ pour tout $F \in \widehat{J \phi}(E)$.

9.3. Corollaire. Soit $\phi$ une fonction non quasi-analytique. Soit $F \in$ $\widehat{J \phi}(E)$. Il existe une fonction $f \in \widehat{\phi}$, réelle analytique sur $\mathbb{R}^{n} \backslash E$, telle que $f=F$ au sens des jets sur $E$.

10. Théorème de Łojasiewicz relatif à une intersection de classes. L'équivalence entre la régulière situation de deux compacts et une propriété de recollement de deux jets définis sur ces compacts a été récemment étudiée par J. Chaumat et A.-M. Chollet dans [3] et par V. Thilliez dans [15]. Dans [3], le théorème de Łojasiewicz est démontré dans le cadre d'intersections de classes non quasi-analytiques à croissance modérée. Dans [15], V. Thilliez démontre l'équivalence entre une régulière situation raffinée et une propriété de recollement avec perte.

Dans tout ce paragraphe les compacts considérés sont inclus dans $\mathbb{R}^{n}$.

\subsection{Généralités}

10.1.1. Définition ([8], [16]). Soient $E_{1}$ et $E_{2}$ deux compacts non vides. On dit que $E_{1}$ et $E_{2}$ sont régulièrement situés s'ils sont disjoints ou s'il existe un ouvert borné $\Omega$ de $\mathbb{R}^{n}$, contenant $E_{1} \cup E_{2}$, et deux constantes $\alpha \geq 1$ et $L>0$ tels que

$$
\forall x \in \Omega, \quad d\left(x, E_{1}\right)+d\left(x, E_{2}\right) \geq \operatorname{Ld}\left(x, E_{1} \cap E_{2}\right)^{\alpha} .
$$

On dira que $E_{1}$ et $E_{2}$ sont $\alpha$-situés.

REMARQUES. (i) Il s'agit de la condition bien connue de régulière situation de Łojasiewicz.

(ii) Quitte à augmenter $\alpha$ on pourra supposer que $\alpha$ est un entier. 
10.1.2. PropriÉté ([8], [16]). Soient $E_{1}$ et $E_{2}$ deux compacts non disjoints. Si $E_{1}$ et $E_{2}$ sont $\alpha$-situés, alors, il existe $\left.\left.D_{1} \in\right] 0,1\right]$ tel que, pour tout $x \in E_{1} \backslash E_{2}$ et tout $\zeta \in E_{2} \backslash E_{1}$, il existe $z \in E_{1} \cap E_{2}$ tel que $|x-\zeta| \geq D_{1}|\zeta-z|^{\alpha}$ et $|x-\zeta| \geq D_{1}|x-z|^{\alpha}$. La constante $D_{1}$ ne dépend que de $E_{1}$ et $E_{2}$.

10.2. ThÉORÈme. Soit $\left(M_{p}\right)_{p \geq 0}$ une suite logarithmiquement convexe vérifiant $M_{0} \leq M_{1}$ et soit $\alpha \in \mathbb{N}^{*}$. Soient $E_{1}$ et $E_{2}$ deux compacts $\alpha$-situés et $a>0$. Soient $F_{1}$ et $F_{2}$ deux jets appartenant respectivement à $\left\{p ! M_{p}, 1, E_{1}\right\}$ et à $\left\{p ! M_{p}, 1, E_{2}\right\}$ et vérifiant $F_{1}=F_{2}$ sur $E_{1} \cap E_{2}$ au sens des jets. Alors le jet $F$ défini par $F(x)=F_{1}(x)$ si $x \in E_{1}$ et $F(x)=F_{2}(x)$ si $x \in E_{2}$ appartient à $\left\{p ! M_{\alpha(p+1)}, A, E_{1} \cup E_{2}\right\}$, où $A$ est une constante ne dépendant que de la géométrie des compacts.

Démonstration. On peut supposer que les compacts ne sont pas disjoints. Soit $J$ un multi-indice de longueur $j \leq p$. Si $x \in E_{1} \backslash E_{2}$ et $\zeta \in E_{2} \backslash E_{1}$, d'après la propriété 10.1.2, il existe $z \in E_{1} \cap E_{2}$ tel que $|x-\zeta| \geq D_{1}|\zeta-z|^{\alpha}$ et $|x-\zeta| \geq D_{1}|x-z|^{\alpha}$. Pour tout $p^{\prime} \geq p$, on a

$$
\begin{aligned}
\left|R_{\zeta}^{J, p} F(x)\right| \leq & \left|R_{\zeta}^{J, p} F(x)-R_{\zeta}^{J, p^{\prime}} F(x)\right| \\
& +\left|R_{\zeta}^{J, p^{\prime}} F(x)-R_{z}^{J, p^{\prime}} F(x)\right|+\left|R_{z}^{J, p^{\prime}} F(x)\right| .
\end{aligned}
$$

(i) Estimons le deuxième terme du membre de droite de (45) :

$$
\left|R_{\zeta}^{J, p^{\prime}} F(x)-R_{z}^{J, p^{\prime}} F(x)\right|=\left|T_{z}^{p^{\prime}-j} F^{J}(x)-T_{\zeta}^{p^{\prime}-j} F^{J}(x)\right| .
$$

Comme dans [16], Ch. 4, p. 69, on a

$$
T_{z}^{p^{\prime}-j} F^{J}(x)-T_{\zeta}^{p^{\prime}-j} F^{J}(x)=\sum_{K ;|K| \leq p^{\prime}-j} \frac{(x-z)^{K}}{K !}\left(R_{\zeta}^{J+K, p^{\prime}} F_{2}\right)(z) .
$$

On note $\left\|F_{2}\right\|=\left\|F_{2}\right\|_{\left\{p ! M_{p}, 1, E_{2}\right\}}$ et on fixe $p^{\prime}=\alpha(p+1)-1$. Si $\delta$ désigne le diamètre de $E_{1} \cup E_{2}$ et $\delta_{1}=\sup (\delta, 1)$, on obtient

$$
\begin{aligned}
& \left|T_{z}^{p^{\prime}-j} F^{J}(x)-T_{\zeta}^{p^{\prime}-j} F^{J}(x)\right| \\
& \quad \leq \sum_{K ;|K| \leq p^{\prime}-j} \frac{\left(\delta_{1} / D_{1}\right)^{p+1}}{K !}\left\|F_{2}\right\|(j+k) ! M_{p^{\prime}+1}|x-\zeta|^{p+1-j} \\
& \quad \leq j ! M_{\alpha(p+1)} \alpha\left(2(2 n)^{\alpha} \frac{\delta_{1}}{D_{1}}\right)^{p+1}\left\|F_{2}\right\||x-\zeta|^{p+1-j} .
\end{aligned}
$$

(ii) Estimons le premier terme du membre de droite de (45) :

$$
\begin{aligned}
& \left|R_{\zeta}^{J, p} F(x)-R_{\zeta}^{J, p^{\prime}} F(x)\right| \\
& \quad \leq \sum_{K ; p<|J+K| \leq p^{\prime}} \frac{1}{K !}|x-\zeta|^{k}\left\|F_{2}\right\|(j+k) ! M_{j+k} \\
& \quad \leq j ! M_{\alpha(p+1)-1}(\alpha-1)\left\|F_{2}\right\|\left(2(2 n)^{\alpha} \delta_{1}^{\alpha-1}\right)^{p+1}|x-\zeta|^{p+1-j} .
\end{aligned}
$$


(iii) Estimons le troisième terme du membre de droite de (45) :

$$
\left|R_{z}^{J, p^{\prime}} F(x)\right| \leq\left\|F_{1}\right\| j ! M_{p^{\prime}+1}|x-z|^{p^{\prime}-j+1},
$$

soit

$$
\left|R_{z}^{J, p^{\prime}} F(x)\right| \leq\left\|F_{1}\right\| j ! M_{\alpha(p+1)} \delta_{1}^{p}\left(1 / D_{1}\right)^{p+1}|x-\zeta|^{p+1-j} .
$$

Les estimations (45)-(48) démontrent le théoréme 10.2 , en posant $A=$ $\left(2 / D_{1}\right)\left(2 n \delta_{1}\right)^{\alpha}$. De plus, on a

$$
\|F\|_{\left\{p ! M_{\alpha(p+1)}, A, E_{1} \cup E_{2}\right\}} \leq 3 \alpha\left(\left\|F_{1}\right\|_{\left\{p ! M_{p}, 1, E_{1}\right\}}+\left\|F_{2}\right\|_{\left\{p ! M_{p}, 1, E_{2}\right\}}\right) .
$$

Remarque. Ici on ne suppose pas que la suite $\left(M_{p}\right)_{p \geq 0}$ est non quasianalytique; la preuve n'utilise pas, contrairement à [3] et à [15], de théorème d'extension de type Whitney.

Par un argument similaire à celui de la fin de la démonstration du théorème 4.1 on établit le théorème de recollement dans $\widehat{J \phi}$ suivant :

10.3. ThÉORÈme. Soit $\alpha \in \mathbb{N}^{*}$ et soient $E_{1}$ et $E_{2}$ deux compacts $\alpha$ situés. Si $F_{1}$ et $F_{2}$ sont deux jets appartenant respectivement à $\widehat{J \phi}\left(E_{1}\right)$, $\widehat{J \phi}\left(E_{2}\right)$ et vérifiant $F_{1}=F_{2}$ sur $E_{1} \cap E_{2}$ au sens des jets, alors le jet $F$ défini par $F(x)=F_{1}(x)$ si $x \in E_{1}$ et $F(x)=F_{2}(x)$ si $x \in E_{2}$ appartient $\grave{a}$ $\widehat{J \phi}\left(E_{1} \cup E_{2}\right)$.

10.4. Théorème RÉCiProque. Soient $E_{1}$ et $E_{2}$ deux compacts. Soit $\phi$ une fonction non quasi-analytique. On suppose que, pour tout couple $\left(F_{1}, F_{2}\right)$ appartenant à $\widehat{J \phi}\left(E_{1}\right) \times \widehat{J \phi}\left(E_{2}\right)$ et vérifiant $F_{1}=F_{2}$ sur $E_{1} \cap E_{2}$ au sens des jets, le jet $F=F_{1} \cup F_{2}$ défini par $F(x)=F_{1}(x)$ si $x \in E_{1}$ et $F(x)=F_{2}(x)$ si $x \in E_{2}$ appartient à $\widehat{J \phi}\left(E_{1} \cup E_{2}\right)$. Alors les compacts $E_{1}$ et $E_{2}$ sont régulièrement situés.

Démonstration. Notons $\widehat{J \phi}\left(E_{1}\right) \diamond \widehat{J \phi}\left(E_{2}\right)$ l'ensemble des couples $\left(F_{1}, F_{2}\right)$ $\in \widehat{J \phi}\left(E_{1}\right) \times \widehat{J \phi}\left(E_{2}\right)$ tels que $F_{1}=F_{2}$ sur $E_{1} \cap E_{2}$ au sens des jets.

Soit $b>0$. D'après le théorème de l'application ouverte entre espaces de Fréchet, il existe deux constantes strictement positives $a$ et $r$ telles que

$$
\begin{aligned}
\forall\left(F_{1}, F_{2}\right) \in \widehat{J \phi}\left(E_{1}\right) \diamond \widehat{J \phi}\left(E_{2}\right), \\
\quad\left\|F_{1} \cup F_{2}\right\|_{\left\{p ! M_{a p}^{(\phi)}, 1, E_{1} \cup E_{2}\right\}} \leq r \sum_{i=1}^{2}\left\|F_{i}\right\|_{\left\{p ! M_{b p}^{(\phi)}, 1, E_{i}\right\}} .
\end{aligned}
$$

Supposons que $E_{1}$ et $E_{2}$ ne soient pas régulièrement situés. D'après la définition 10.1.1 il existe une suite $\left(x_{j}\right)_{j \geq 0}$ d'éléments de $E_{1}$ telle que

$$
\forall j \in \mathbb{N}, \quad 0<d\left(x_{j}, E_{2}\right) \leq\left(d\left(x_{j}, E_{1} \cap E_{2}\right)\right)^{j} \quad \text { et } \quad d\left(x_{j}, E_{1} \cap E_{2}\right) \leq 1 / 2 .
$$

Pour tout $j \in \mathbb{N}$, on pose $r_{j}=d\left(x_{j}, E_{1} \cap E_{2}\right)$ et on note $B_{j}$ la boule $B\left(x_{j}, r_{j} / 2\right)$. En utilisant la proposition 4.2 avec $u_{p}=1$ on établit, comme 
au paragraphe 3.1.3, l'existence d'une constante $A>0$ et de fonctions $\phi_{j} \in$ $\left\{p ! M_{p}^{(\psi)}, A / r_{j}, \mathbb{R}^{n}\right\}, j \in \mathbb{N}$, telles que $\phi_{j}\left(x_{j}\right)=1$ et $\operatorname{supp}\left(\phi_{j}\right) \subset B_{j}$. Soit $\Phi_{j}=R_{E_{1}}\left(\phi_{j}\right), \Phi_{j} \in\left\{p ! M_{p}^{(\psi)}, A^{\prime} / r_{j}, E_{1}\right\}$, où $A^{\prime}$ est une constante supérieure ou égale à 1 ne dépendant que de la suite $\left(M_{p}^{(\psi)}\right)_{p \geq 0}$. Donc

$$
\Phi_{j} \in \widehat{J \phi}\left(E_{1}\right)
$$

et, de plus,

$$
\forall b>0, \quad\left\|\Phi_{j}\right\|_{\left\{p ! M_{b p}^{(\phi)}, 1, E_{1}\right\}} \leq M_{0}^{(\phi)} C_{2}\left(\frac{b}{2}\right) \sup _{k \in \mathbb{N}}\left(\frac{1}{M_{b k / 2}^{(\phi)}}\left(\frac{A^{\prime}}{r_{j}}\right)^{k}\right) .
$$

Soit $y_{j}$ un élément de $E_{2}$ réalisant la distance $d\left(x_{j}, E_{2}\right)$. En utilisant (50) on a

$$
\forall p \in \mathbb{N}, \quad\left|R_{y_{j}}^{0, p}\left(\Phi_{j} \cup 0\right)\left(x_{j}\right)\right|=1 \leq\left\|\Phi_{j} \cup 0\right\|_{\left\{p ! M_{a p}^{(\phi)}, 1, E_{1} \cup E_{2}\right\}} M_{a(p+1)}^{(\phi)}\left(r_{j}^{j}\right)^{p+1} .
$$

En utilisant (49) et (51), on obtient

$$
\forall p \in \mathbb{N}, \quad 1 \leq r M_{0}^{(\phi)} C_{2}\left(\frac{b}{2}\right) \sup _{k \in \mathbb{N}}\left(\frac{1}{M_{b k / 2}^{(\phi)}}\left(\frac{A^{\prime}}{r_{j}}\right)^{k}\right) M_{a(p+1)}^{(\phi)} r_{j}^{j(p+1)} .
$$

Soit $k_{0}(j)$ un entier réalisant cette borne supérieure. On a

$$
\forall p \in \mathbb{N}, \quad 1 \leq r M_{0}^{(\phi)} C_{2}\left(\frac{b}{2}\right)\left(\frac{1}{M_{b k_{0}(j) / 2}^{(\phi)}}\left(\frac{A^{\prime}}{r_{j}}\right)^{k_{0}(j)}\right) M_{a(p+1)}^{(\phi)} r_{j}^{j(p+1)} .
$$

Si $k_{0}(j) \leq j$ à partir d'un certain rang, alors, avec $p=1$, (52) implique

$$
1 \leq r C_{2}\left(\frac{b}{2}\right)\left(\left(\frac{A^{\prime}}{r_{j}}\right)^{j}\right) M_{2 a}^{(\phi)} r_{j}^{2 j}=r C_{2}\left(\frac{b}{2}\right)\left(A^{\prime} r_{j}\right)^{j} M_{2 a}^{(\phi)}
$$

pour $j$ assez grand, ce qui est absurde lorsque $j$ tend vers $+\infty$. On peut donc supposer $k_{0}(j)>j$ pour une infinité d'entiers $j$. On note $E(t)$ la partie entière de $t$, pour tout $t \in \mathbb{R}$. En choisissant $p=E\left(b k_{0}(j) /(2 a)\right)-2$, on a $a(p+1) \leq b k_{0}(j) / 2$ et $(52)$ implique

$$
1 \leq r M_{0}^{(\phi)} C_{2}\left(\frac{b}{2}\right)\left(A^{\prime}\right)^{k_{0}(j)} r_{j}^{j\left(E\left(b k_{0}(j) /(2 a)\right)-1\right)-k_{0}(j)} .
$$

Comme

$$
j\left(E\left(\frac{b k_{0}(j)}{2 a}\right)-1\right) \underset{j \rightarrow+\infty}{\sim} j \frac{b k_{0}(j)}{2 a}
$$

et comme $k_{0}(j)$ est négligeable devant $j b k_{0}(j) /(2 a)$, l'inégalité précédente est impossible pour $j$ assez grand, ce qui démontre le théorème 10.4. 


\section{Bibliographie}

[1] J. Chaumat et A.-M. Chollet, Théorème de Whitney dans des classes ultradifférentiables, Publ. Inst. Rech. Math. Lille 28 (1992), VIII.1-VIII.32 et C. R. Acad. Sci. Paris Sér. I 315 (1992), 901-906.

[2] —, - Surjectivité de l'application restriction à un compact dans des classes de fonctions ultradifférentiables, Math. Ann. 298 (1994), 7-40.

[3] —, - Propriétés de l'intersection des classes de Gevrey et de certaines autres classes, Bull. Sci. Math. 122 (1998), 455-485.

[4] -, - Sur la division et la composition dans des classes ultradifférentiables, Studia Math. 136 (1999), 49-79.

[5] R. Coifman et G. Weiss, Analyse harmonique non commutative sur certains espaces homogènes, Lecture Notes in Math. 242, Springer, 1972.

[6] U. Franken, Extension of functions with $\omega$-rapid polynomial approximation, J. Approx. Theory 82 (1995), 88-98.

[7] L. Hörmander, The Analysis of Partial Differantial Operators I, Springer, 1989.

[8] B. Malgrange, Ideals of Differentiable Functions, Oxford Univ. Press, 1966.

[9] W. Pawłucki and W. Pleśniak, Extension of $C^{\infty}$ functions from sets with polynomial cusps, Studia Math. 88 (1988), 279-287.

[10] H.-J. Petzsche, On E. Borel's theorem, Math. Ann. 282 (1988), 299-313.

[11] W. Pleśniak, Extension and polynomial approximation of ultradifferentaible functions in $\mathbb{R}^{N}$, Bull. Soc. Roy. Sci. Liège 63 (1994), 393-402.

[12] - Markov's inequality and the existence of an extension operator for $C^{\infty}$ functions, J. Approx. Theory 61 (1990), 106-117.

[13] - Recent progress in multivariate Markov inequality, in: Approximation Theory. In memory of A. K. Varma, Pure Appl. Math. 212, Marcel Dekker, 1998, 449-464.

[14] J. Siciak, Extremal plurisubharmonic functions in $\mathbf{C}^{n}$, Ann. Polon. Math. 39 (1981), $175-211$.

[15] V. Thilliez, Prolongement dans des classes ultradifférentiables et propriétés de régularité des compacts de $\mathbb{R}^{n}$, ibid. 63 (1996), 71-88.

[16] J.-C. Tougeron, Idéaux de fonctions différentiables, Springer, 1972.

[17] M. Valdivia, On certain analytic function ranged linear operators in spaces of ultradifferentiable functions, Math. Japon. 44 (1996), 415-434.

[18] H. Whitney, Analytic extensions of differentiable functions defined in closed sets, Trans. Amer. Math. Soc. 36 (1934), 63-89.

Mathématiques

Université de Paris-Sud

Bâtiment 425

91405 Orsay, France

E-mail: pbeaugendre@ifrance.com

Pascal.Beaugendre@math.u-psud.fr 